\title{
A new approach to gauge coupling unification and proton decay
}

\author{
Stefan Pokorski, ${ }^{a}$ Krzysztof Rolbiecki, ${ }^{a}$ Graham G. Ross ${ }^{b}$ and Kazuki Sakurai ${ }^{a}$ \\ ${ }^{a}$ Institute of Theoretical Physics, Faculty of Physics, University of Warsaw, \\ Pasteura 5, PL-02-093 Warsaw, Poland \\ ${ }^{b}$ Rudolf Peierls Centre for Theoretical Physics, Clarendon Laboratory, University of Oxford, \\ Park Roads, Oxford OX1 3PU, U.K. \\ E-mail: Stefan.Pokorski@fuw.edu.pl, krzysztof.rolbiecki@fuw.edu.pl, \\ graham.ross@physics.ox.ac.uk, kazuki.sakurai@fuw.edu.pl
}

AbStRaCt: An analytical formalism, including RG running at two loop order, is used to link the supersymmetric and GUT spectra in any GUT model in which the three gauge couplings unify. In each specific GUT model, one can then fully explore the interplay between the pattern of supersymmetry breaking and the prediction for the proton lifetime. With this formalism at hand, we study three concrete GUT models: (i) Minimal SU(5) SUSY GUT, (ii) Missing Partner SU(5) SUSY GUT, and (iii) an orbifold SU(5) SUSY GUT. In each case we derive interesting conclusions about the possible patterns of the supersymmetric spectrum once the present limits on the proton lifetime are imposed, and vice versa, we obtain the predictions for the proton lifetime for specific viable choices of the SUSY spectrum.

KeYwords: Supersymmetry Phenomenology

ARXIV EPRINT: 1902.06093 


\section{Contents}

1 Introduction 1

2 The formalism 2

3 Minimal SU(5) 5

4 Missing partner SU(5) models $\quad 9$

4.1 Hagiwara-Yamada model 9

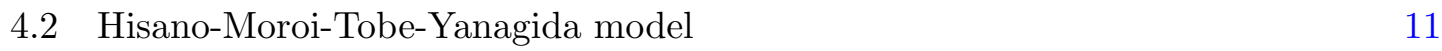

$\begin{array}{lll}5 & \text { SU(5) orbifold SUSY GUT } & 13\end{array}$

$\begin{array}{llr}6 & \text { Discussions and conclusions } & 18\end{array}$

$\begin{array}{lr}\text { A Interpretations of threshold corrections } & 19\end{array}$

A.1 Mass independent unified gauge coupling 20

$\begin{array}{ll}\text { A.2 Sequential matchings } & 21\end{array}$

A.3 A comment on orbifold GUTs 22

\section{Introduction}

The idea of embedding the gauge symmetry groups of the Standard Model (SM) into a larger symmetry, unifying the elementary forces of the SM, is very attractive [1]. Remarkably, the fermion spectrum of the SM nicely fits into the multiplets of the simplest Grand Unification symmetry groups, $\mathrm{SU}(5)$ and $\mathrm{SO}(10)$. Moreover, these symmetry groups predict that the SM gauge couplings are related to the single underlying GUT gauge coupling for some choice of the superheavy GUT spectrum. It has been claimed as a big success of supersymmetry (SUSY) that the gauge couplings of the SM do unify in the Minimal Supersymmetric Standard Model (MSSM), in the presence of SUSY threshold corrections at $\mathcal{O}(1) \mathrm{TeV}$ and with negligible GUT scale threshold corrections, with the superheavy GUT states having mass at the scale $\sim 10^{16} \mathrm{GeV}$, high enough to sufficiently suppress $D=6$ operator contribution to proton decay.

However, quantitative analyses in concrete GUT models are much more demanding and model dependent. Generically, neither the GUT threshold corrections are negligible nor the SUSY spectrum is expected to be degenerate. Constraints from non-observation of proton decay are also model dependent. Among possible decay channels, a special and universal role is played by the $p \rightarrow \pi^{0} e^{+}$mode for which the dominant contribution comes from the $D=6$ operators depending almost exclusively on the $X, Y$ gauge boson mass and the value of the unified gauge coupling. In general the $p \rightarrow K^{+} \nu$ mode, induced by 
the $D=5$ operators generated by the coloured Higgs exchange diagrams, may also give a strong constraint on the colour triplet Higgs mass and low energy SUSY spectrum as well as the structure of the Higgs sector in the GUT models.However, this mode is highly model-dependent and several mechanisms have been constructed to eliminate the $D=5$ operator contribution.

The gauge coupling unification implies a non-trivial relation between SUSY and GUT spectra, which may lead to an interesting interplay between the signatures at collider and proton decay experiments. A pioneering work has been carried out in refs. [2, 3], where an analytical formula relating SUSY and GUT spectra have been derived at one-loop level in the minimal SU(5) SUSY GUT model, with two-loop running included numerically. Similar one-loop formulae have also been presented for the models proposed in [4] and [5]. The interplay of the SUSY and GUT threshold corrections in the unification of the gauge couplings in the MSSM has been studied in various contexts, mostly by numerical methods [6-20]. Most such works have focused on constraining the GUT spectra, assuming the low energy supersymmetric spectrum is degenerate at $m_{\mathrm{SUSY}}$. In this paper we propose to use an analytical formalism linking the SUSY and GUT spectra based on the parametrization of the one-loop SUSY and GUT threshold corrections in terms of three effective parameters describing each of them. ${ }^{1}$ The link between the two spectra necessary to ensure the gauge coupling unification at two-loop level can then be expressed as two relations between two pairs of these parameters. The effective parameters are calculable in terms of particle masses. The formalism provides a convenient way to analyse general GUT models with arbitrary SUSY spectra. In each specific GUT model, one can then fully explore the interplay between the pattern of supersymmetry breaking and the prediction for the proton lifetime. We apply this formalism to four concrete examples of the GUT models. In each case we derive interesting conclusions about the possible patterns of the supersymmetric spectrum once the present limits on the proton lifetime are imposed, and vice versa, we obtain the predictions for the proton lifetime for specific viable choices of the SUSY spectrum.

\section{The formalism}

In this paper we assume that there is an underlying simple GUT group unifying the SM gauge couplings. The relation of the gauge couplings at $m_{Z}$ to the universal gauge coupling evaluated at a scale $\Lambda$, of order the superheavy masses, is given by solving the renormalization group equations (RGEs). The solution can be written as [21, 24-26]

$$
\frac{2 \pi}{\alpha(\Lambda)}=\frac{2 \pi}{\alpha_{i}\left(m_{Z}\right)}-b_{i} \ln \frac{\Lambda}{m_{Z}}+s_{i}+r_{i}+\gamma_{i}+\Delta_{i}
$$

where $\alpha_{1} \equiv \frac{5}{3} \alpha_{Y}, i=1,2,3$, represents the gauge group and $b_{i}=\left(\frac{33}{5}, 1,-3\right)$ are the oneloop $\beta$-function coefficients for the MSSM. The quantities $s_{i}$ and $r_{i}$ represent the low energy supersymmetric and GUT scale threshold corrections, respectively. (See the appendix for

\footnotetext{
${ }^{1}$ Similar parametrizations of SUSY spectra have been previously used in refs. [21-23].
} 
the derivation and interpretations of the threshold corrections.) They read

$$
s_{i}=\sum_{\eta} b_{i}^{\eta} \ln \frac{m_{\eta}}{m_{Z}}
$$

and

$$
r_{i}=\sum_{\xi} b_{i}^{\xi} \ln \frac{m_{\xi}}{\Lambda}
$$

The parameters $m_{\eta}$ and $b_{i}^{\eta}$ denote the mass and the contribution to $b_{i}$ from the superparticle $\eta$. The parameters $m_{\xi}$ and $b_{i}^{\xi}$ are the corresponding parameters for the GUT scale particle $\xi$. Both spectra are arbitrary at this point. Clearly, gauge coupling unification puts strong constraints on the sums $s_{i}+r_{i}$ that we are going to quantify in the following. The $\gamma_{i} \equiv-\frac{1}{2} \sum_{i} \frac{b_{i j}}{b_{i}} \ln \left(\frac{\alpha_{j}(\Lambda)}{\alpha_{j}\left(m_{Z}\right)}\right)$ accounts for the two-loop contribution with $b_{i j}$ being the twoloop $\beta$-function coefficients. ${ }^{2}$ The $\Delta_{i}$ represents the effect of the top Yukawa coupling and the conversion factor between $\overline{M S}$ and $\overline{D R}$ schemes. ${ }^{3}$

Since any 3-dimensional vector can be expanded in terms of 3 independent vectors, the three terms in eq. $(2.1),\left[\left(2 \pi \alpha^{-1}\left(m_{Z}\right)+\gamma+\Delta\right)_{i}, s_{i}, r_{i}\right]$, can be expanded in terms of $\left[(1,1,1), b_{i}, \delta_{i}\right]$ as

$$
\begin{aligned}
\frac{2 \pi}{\alpha_{i}\left(m_{Z}\right)} & =\frac{2 \pi}{\alpha_{G}^{*}}+b_{i} \ln \frac{M_{G}^{*}}{m_{Z}}-\delta_{i} \ln \frac{M_{S}^{*}}{m_{Z}}-\gamma_{i}-\Delta_{i}, \\
s_{i} & =C_{S}+b_{i} \ln \Omega_{S}+\delta_{i} \ln \frac{T_{S}}{m_{Z}}, \\
r_{i} & =C_{G}-b_{i} \ln \frac{T_{G}}{\Lambda}-\delta_{i} \ln \Omega_{G},
\end{aligned}
$$

where $b_{i}$ is the MSSM $\beta$-function coefficients and $\delta_{i} \equiv b_{i}-b_{i}^{\mathrm{SM}}$ is the difference between those and the SM ones. The parameters $C_{S}, T_{S}, \Omega_{S}$ and $C_{G}, T_{G}, \Omega_{G}$ fully parametrize any arbitrary supersymmetric and GUT threshold corrections at the leading logarithmic level. They can be found by solving the second and third set of the equations, once $s_{i}$ and $r_{i}$ are given in terms of concrete spectra.

The first set of the above equations can be interpreted as the solution to the RGE for the special case where the GUT threshold correction is absent and all SUSY particles are degenerate at $M_{S}^{*}$. The $M_{G}^{*}$ and $\alpha_{G}^{*-1}$ are then the unification scale and the unified coupling for this idealised situation, respectively. We find numerically that $\alpha_{G}^{*-1}=25.5$, $M_{G}^{*}=1.26 \cdot 10^{16} \mathrm{GeV}, M_{S}^{*}=2.13 \mathrm{TeV}$ solve the first set of equations for the experimental values of the gauge couplings at $m_{Z}$ with the recent world average $\alpha_{s}^{0}\left(m_{Z}\right)=0.1183[27]$. By varying the $\alpha_{s}\left(m_{Z}\right)$ within its $1-\sigma$ error, $\Delta \alpha_{s}=0.0008$ [27], we have $M_{S}^{*} \in[2.69,1.72] \mathrm{TeV}$, $M_{G}^{*} \in[1.17,1.35] \cdot 10^{16} \mathrm{GeV}$ and $\alpha_{G}^{*} \in[25.7,25.4]$, where the left and right values correspond to the negative and positive variation of the strong coupling $\alpha_{s}\left(m_{Z}\right) \in[0.1175,0.1191]$. For

\footnotetext{
${ }^{2}$ At the scale $\Lambda$, it can be approximated as $\gamma_{i}=\frac{1}{4 \pi} \sum_{i} \frac{b_{i j}}{b_{i}} \ln \left(1+\frac{b_{j} \alpha_{\Lambda}}{2 \pi} \ln \frac{\Lambda}{m_{Z}}\right)$. One can solve $\gamma_{i}$ iteratively by updating $\Lambda$ and $\alpha_{\Lambda}$ [21].

${ }^{3}$ For the treatment of $\Delta_{i}$, see for example [21].
} 
more general cases, those constants can be approximately written in terms of $\alpha_{s}\left(m_{Z}\right)$ as

$$
\begin{aligned}
\frac{M_{S}^{*}}{\mathrm{TeV}} & =\frac{2.13}{\mathrm{TeV}} \cdot \exp \left[-0.224\left(\frac{\alpha_{s}-\alpha_{s}^{0}}{\Delta \alpha_{s}}\right)\right], \\
\frac{M_{G}^{*}}{\mathrm{GeV}} & =\frac{1.26 \cdot 10^{16}}{\mathrm{GeV}} \cdot \exp \left[0.0715\left(\frac{\alpha_{s}-\alpha_{s}^{0}}{\Delta \alpha_{s}}\right)\right], \\
\alpha_{G}^{*-1} & =25.5-0.172\left(\frac{\alpha_{s}-\alpha_{s}^{0}}{\Delta \alpha_{s}}\right) .
\end{aligned}
$$

It is convenient to trade the three experimental numbers, $\alpha_{i}\left(m_{Z}\right)$, for the three new parameters $\alpha_{G}^{*}, M_{G}^{*}, M_{S}^{*}$. Substituting eq. (2.4) into eq. (2.1), we get

$$
\frac{2 \pi}{\alpha(\Lambda)}=\left[\frac{2 \pi}{\alpha_{G}^{*}}+C_{S}+C_{G}\right]+b_{i} \ln \left(\frac{M_{G}^{*} \Omega_{S}}{T_{G}}\right)+\delta_{i} \ln \left(\frac{T_{S}}{M_{S}^{*} \Omega_{G}}\right) .
$$

Since the three basis-vectors of this expansion are independent, in order for the left-handside to be $i$-independent the two logarithms in the right-hand-side must vanish. This is the condition of the gauge coupling unification. Namely, the GUT and SUSY spectra must satisfy the following simultaneous conditions:

$$
T_{S}=M_{S}^{*} \Omega_{G} \quad \cap \quad T_{G}=M_{G}^{*} \Omega_{S} .
$$

The inverse of unified gauge coupling is then given by:

$$
\alpha^{-1}(\Lambda)=\alpha_{G}^{*-1}+\frac{C_{S}+C_{G}}{2 \pi}
$$

The second and third equations in eq. (2.4) can easily be solved. The general solutions can be written as

$$
\begin{aligned}
\ln \frac{T_{S}}{m_{Z}} & =v_{i} s_{i} / D, \\
\ln \Omega_{S} & =u_{i} s_{i} / D, \\
C_{S} & =-\epsilon_{i j k} b_{i} \delta_{j} s_{k} / D, \\
\ln \Omega_{G} & =-v_{i} r_{i} / D, \\
\ln \frac{T_{G}}{\Lambda} & =-u_{i} r_{i} / D, \\
C_{G} & =-\epsilon_{i j k} b_{i} \delta_{j} r_{k} / D,
\end{aligned}
$$

where

$$
\begin{aligned}
& v=\left(\begin{array}{c}
b_{2}-b_{3} \\
-b_{1}+b_{3} \\
b_{1}-b_{2}
\end{array}\right)=\left(\begin{array}{c}
4 \\
-\frac{48}{5} \\
\frac{28}{5}
\end{array}\right), \quad u=\left(\begin{array}{c}
-\delta_{2}+\delta_{3} \\
\delta_{1}-\delta_{3} \\
-\delta_{1}+\delta_{2}
\end{array}\right)=\left(\begin{array}{c}
-\frac{1}{6} \\
-\frac{3}{2} \\
\frac{5}{3}
\end{array}\right), \\
& D=b_{2} \delta_{1}-b_{3} \delta_{1}-b_{1} \delta_{2}+b_{3} \delta_{2}+b_{1} \delta_{3}-b_{2} \delta_{3}=-\frac{38}{5},
\end{aligned}
$$


where we used $b_{i}=\left(\frac{33}{5}, 1,-3\right), b_{i}^{\mathrm{SM}}=\left(\frac{41}{10},-\frac{19}{6},-7\right)$ and $\delta_{i}=b_{i}-b_{i}^{\mathrm{SM}}=\left(\frac{5}{2}, \frac{25}{6}, 4\right)$. Substituting these numbers the solutions become [28]

$$
\begin{aligned}
T_{S}= & {\left[M_{3}^{-28} M_{2}^{32} \mu^{12} m_{A}^{3} X_{T}\right]^{\frac{1}{19}}, } \\
\Omega_{S}= & {\left[M_{3}^{-100} M_{2}^{60} \mu^{32} m_{A}^{8} X_{\Omega}\right]^{\frac{1}{288}}, } \\
C_{S}= & \frac{125}{19} \ln M_{3}-\frac{113}{19} \ln M_{2}-\frac{40}{19} \ln \mu-\frac{10}{19} \ln m_{A} \\
& +\sum_{i=1 \ldots 3}\left[\frac{79}{114} \ln m_{\tilde{d}_{R i}}-\frac{10}{19} \ln m_{\tilde{l}_{i}}-\frac{121}{114} \ln m_{\tilde{q}_{i}}+\frac{257}{228} \ln m_{\tilde{u}_{R i}}+\frac{33}{76} \ln m_{\tilde{e}_{R i}}\right],
\end{aligned}
$$

with

$$
X_{T} \equiv \prod_{i=1 \ldots 3}\left(\frac{m_{\tilde{l}_{i}}^{3}}{m_{\tilde{d}_{R i}}^{3}}\right)\left(\frac{m_{\tilde{q}_{i}}^{7}}{m_{\tilde{e}_{R i}}^{2} m_{\tilde{u}_{R i}}^{5}}\right), \quad X_{\Omega} \equiv \prod_{i=1 \ldots 3}\left(\frac{m_{\tilde{l}_{i}}^{8}}{m_{\tilde{d}_{R i}}^{8}}\right)\left(\frac{m_{\tilde{q}_{i}}^{6} m_{\tilde{e}_{R i}}}{m_{\tilde{u}_{R i}}^{7}}\right),
$$

for the MSSM sparticles and

$$
\begin{aligned}
\ln \Omega_{G} & =\sum_{\xi}\left(\frac{10}{19} b_{1}^{\xi}-\frac{24}{19} b_{2}^{\xi}+\frac{14}{19} b_{3}^{\xi}\right) \ln \frac{m_{\xi}}{\Lambda}, \\
\ln \frac{T_{G}}{\Lambda} & =\sum_{\xi}\left(-\frac{5}{228} b_{1}^{\xi}-\frac{15}{76} b_{2}^{\xi}+\frac{25}{114} b_{3}^{\xi}\right) \ln \frac{m_{\xi}}{\Lambda}, \\
C_{G} & =\sum_{\xi}\left(\frac{165}{76} b_{1}^{\xi}-\frac{339}{76} b_{2}^{\xi}+\frac{125}{38} b_{3}^{\xi}\right) \ln \frac{m_{\xi}}{\Lambda},
\end{aligned}
$$

for the GUT scale particles. In eq. (2.11) the parameters $M_{2}, M_{3}, m_{A}$ and $\mu$ denote the wino, gluino, the CP-odd scalar soft masses and the higgsino mass, respectively, each at the corresponding decoupling (threshold) scale.

The condition eq. (2.7) with eqs. (2.11) and (2.13) have to be satisfied for arbitrary supersymmetric and GUT scale spectra to ensure the gauge coupling unification. These compact relations can be used, for instance, to discuss the patterns of the supersymmetric spectra consistent with the unification in various concrete GUT models; e.g. conventional models in $4 \mathrm{~d}$ or in extra dimensional models, with the present and future limits on the proton decay imposed. They quantify a non-trivial interconnection between collider and proton decay experiments. In the following we shall discuss the implications of the gauge coupling unification, i.e. eq. (2.7), in several concrete GUT models.

\section{Minimal SU(5)}

The first example in which we apply our formula and study an interplay between the low energy SUSY and GUT spectra is the minimal SU(5) model. The Higgs sector of this model contains the adjoint chiral multiplet $\Sigma(\mathbf{2 4})=\left(\Sigma_{8}, \Sigma_{(2,3)}, \Sigma_{(2, \overline{3})}, \Sigma_{3}, \Sigma_{1}\right)$ and the (anti-)fundamental chiral multiplet $H(\mathbf{5})=\left(H_{C}, H_{u}\right)\left(\bar{H}(\overline{\mathbf{5}})=\left(\bar{H}_{C}, \epsilon H_{d}\right)\right)$. In the above notation, $\Sigma_{i}$ are the component of $\Sigma$ under $G_{\mathrm{SM}}=\mathrm{U}(1) \times \mathrm{SU}(2) \times \mathrm{SU}(3)$ decomposition, 


\begin{tabular}{|c|c|c|}
\hline mass & $(\mathrm{U}(1) \times \mathrm{SU}(2) \times \mathrm{SU}(3))$ & $\left(b_{1}, b_{2}, b_{3}\right)$ \\
\hline$M_{H_{C}}$ & $\left(-\frac{1}{3}, \mathbf{1}, \mathbf{3}\right),\left(\frac{1}{3}, \mathbf{1}, \overline{\mathbf{3}}\right)$ & $\left(\frac{2}{5}, 0,1\right)$ \\
\hline$M_{V}$ & $\left(-\frac{5}{6}, \mathbf{2}, \mathbf{3}\right),\left(\frac{5}{6}, \mathbf{2}, \overline{\mathbf{3}}\right)$ & $(-10,-6,-4)$ \\
\hline$M_{\Sigma}$ & $(0, \mathbf{3}, \mathbf{1}),(0, \mathbf{1}, \mathbf{8})$ & $(0,2,3)$ \\
\hline
\end{tabular}

Table 1. The GUT mass spectrum and the contribution to the $\beta$-function coefficients in the Minimal SU(5) model.

$H_{C}\left(\bar{H}_{C}\right)$ is the colour (anti-)triplet Higgs field and $H_{u / d}$ are the doublet Higgs fields in the MSSM. The Higgs superpotential is given by

$$
W_{H}=\frac{1}{2} M \operatorname{Tr} \Sigma^{2}+\frac{1}{3} \lambda_{\Sigma} \operatorname{Tr} \Sigma^{3}+\lambda_{H} \bar{H}(\Sigma+3 V) \Sigma H,
$$

where the dimensionfull parameter $V$ is related to the VEV of $\Sigma$ as

$$
\langle\Sigma\rangle=V \cdot \operatorname{diag}(2,2,2,-3,-3),
$$

such that the MSSM Higgs fields become massless by the cancellation in the last term of eq. (3.1). This cancellation is called doublet-triplet splitting problem since it requires an enormous fine-tuning. The mass of colour triplet Higgses reads

$$
M_{H_{C}}=5 \lambda_{H} V
$$

The direction of the VEV in eq. (3.2) breaks the SU(5) gauge symmetry to $G_{\mathrm{SM}}$, giving masses to the $X, Y$ gauge bosons

$$
M_{V}=5 \sqrt{2} g_{5} V,
$$

with $g_{5}$ being the $\mathrm{SU}(5)$ gauge coupling. While the Goldstone components $\Sigma_{(3,2)}, \Sigma_{(\overline{3}, 2)}$ in the $\Sigma$ field are eaten by the $X, Y$ fields, the $\Sigma_{8}$ and $\Sigma_{3}$ components have the mass

$$
M_{\Sigma}=\frac{5}{2} M
$$

and contribute to the GUT threshold correction together with the $X, Y$ and $H_{C}, \bar{H}_{C}$ fields. The singlet field $\Sigma_{1}$ has the mass $\frac{1}{2} M$ but does not contribute to the GUT threshold correction, thus is irrelevant to our discussion.

We summarise the field components that contribute to the GUT threshold correction in table 1. By plugging the masses and $\beta$-function coefficients into the formula (2.13), we find

$$
\begin{aligned}
\Omega_{G} & =M_{H_{C}}^{\frac{18}{19}} M_{V}^{-\frac{12}{19}} M_{\Sigma}^{-\frac{6}{19}}, \\
T_{G} & =M_{H_{C}}^{\frac{4}{19}} M_{V}^{\frac{10}{19}} M_{\Sigma}^{\frac{5}{19}}, \\
C_{G} & =3 \ln \frac{\Lambda}{M_{G}^{*}}+\frac{79}{19} \ln \frac{M_{H_{C}}}{M_{G}^{*}}-\frac{154}{19} \ln \frac{M_{V}}{M_{G}^{*}}+\frac{18}{19} \ln \frac{M_{\Sigma}}{M_{G}^{*}},
\end{aligned}
$$



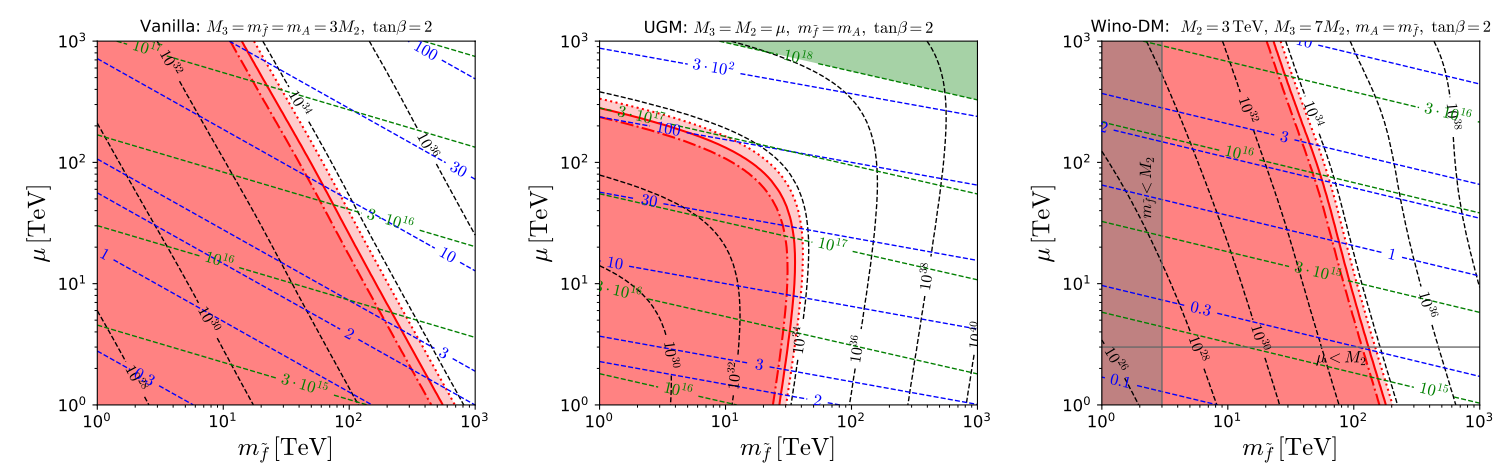

Figure 1. The low energy SUSY parameter space $\left(m_{\tilde{f}}\right.$ versus $\mu$ plane) for three different scenarios: Left; "vanilla" SUSY with $M_{3}=3 M_{2}=m_{A}=m_{\tilde{f}}$ and $\tan \beta=2$. Centre; UGM scenario with $M_{3}=M_{2}=\mu, m_{A}=m_{\tilde{f}}$ and $\tan \beta=2$. Right; Wino DM scenario with $M_{2}=3 \mathrm{TeV}$, $M_{3}=7 M_{2}, m_{A}=m_{\tilde{f}}$ and $\tan \beta=2$. The light red region is excluded due to the current limit, $\tau\left(p \rightarrow K^{+} \bar{\nu}\right)>4.0 \cdot 10^{33}$ yrs. The band around the boundary of the red region represents the uncertainty coming from $\alpha_{s}\left(m_{Z}\right)$. The dashed-dotted and dotted contours correspond to the limit obtained from $\alpha_{s}\left(m_{Z}\right)=\alpha_{s}^{0} \pm \Delta \alpha_{s}$, respectively. The dashed black, green and blue contours represent the values of $\tau\left(p \rightarrow K^{+} \bar{\nu}\right) / \mathrm{yrs}, M_{H_{C}} / \mathrm{GeV}$ and $T_{S} / \mathrm{TeV}$, respectively. The shaded green region is disfavoured because $M_{H_{C}}$ is close to the Planck scale.

where we have used $b^{G}=(-3,-3,-3)$ for the full Minimal SU(5) $\beta$-function coefficients. Using the unification conditions eq. (2.7), one obtains

$$
\begin{aligned}
M_{H_{C}} & =M_{G}^{*} \Omega_{S}\left(\frac{T_{S}}{M_{S}^{*}}\right)^{\frac{5}{6}} \\
\left(M_{V}^{2} M_{\Sigma}\right)^{\frac{1}{3}} & =M_{G}^{*} \Omega_{S}\left(\frac{T_{S}}{M_{S}^{*}}\right)^{-\frac{2}{9}} .
\end{aligned}
$$

It is worth noting that despite the look of eq. (2.13), these conditions do not depend on $\Lambda$. A general proof of the $\Lambda$ independence and an exceptional case are given and discussed in the appendix. The above equations are remarkable in the sense that they allow us to analytically calculate the masses of superheavy particles in terms of the low energy SUSY spectrum through $T_{S}$ and $\Omega_{S}$ given in eq. (2.11).

Eq. (3.7) is particularly interesting since it allows the prediction of the $D=5$ proton decay rate purely from the low energy SUSY spectrum. ${ }^{4}$ The results are plotted in figure 1 in the $\left(m_{\tilde{f}}, \mu\right)$ SUSY plane, where $m_{\tilde{f}}$ is the universal sfermion mass, for three scenarios: (Left; "Vanilla" SUSY, Centre; Universal Gaugino Mass (UGM) scenario, Right; Wino DM scenario. The details of these scenarios are given at the end of this section.)

In the plots, the light red region is excluded by the current proton decay limit $\tau\left(p \rightarrow K^{+} \bar{\nu}\right)>4.0 \cdot 10^{33}$ yrs. The band around the boundary of the red region represents the uncertainty coming from $\alpha_{s}\left(m_{Z}\right)$. The dashed-dotted and dotted contours correspond to the limit obtained from the upper and lower 1- $\sigma$ variations of $\alpha_{s}\left(m_{Z}\right)$, respectively. The

\footnotetext{
${ }^{4}$ In the calculation of the $D=5$ proton decay, we closely follow ref. [13]. We thank J. Hisano and N. Nagata for the details of the calculation.
} 
dashed black, green and blue contours represent the values of $\tau\left(p \rightarrow K^{+} \bar{\nu}\right) / \mathrm{yrs}, M_{H_{C}} / \mathrm{GeV}$ and $T_{S} / \mathrm{TeV}$, respectively. The shaded green region is disfavoured because there $M_{H_{C}}$ is very close to the Planck scale.

We also compute $\left(M_{V}^{2} M_{\Sigma}\right)^{\frac{1}{3}}$ and the unified coupling $\alpha^{-1}(\Lambda)$ for $\Lambda=\max \left\{M_{H_{C}}, M_{V}\right\}$, assuming $M_{V}=M_{\Sigma}$, for our three example scenarios. We found that $\left(M_{V}^{2} M_{\Sigma}\right)^{\frac{1}{3}}$ varies very mildly due to the small power in eq. (3.8). Over the region shown on the plots we find:

$$
\begin{aligned}
\text { Vanilla SUSY : } & 3.77 \cdot 10^{15} \mathrm{GeV}<\left(M_{V}^{2} M_{\Sigma}\right)^{\frac{1}{3}}<1.83 \cdot 10^{16} \mathrm{GeV}, \\
\mathrm{UGM}: & 3.14 \cdot 10^{15} \mathrm{GeV}<\left(M_{V}^{2} M_{\Sigma}\right)^{\frac{1}{3}}<1.52 \cdot 10^{16} \mathrm{GeV} \\
\text { Wino DM : } & 8.90 \cdot 10^{15} \mathrm{GeV}<\left(M_{V}^{2} M_{\Sigma}\right)^{\frac{1}{3}}<1.19 \cdot 10^{16} \mathrm{GeV}
\end{aligned}
$$

Generally, light $X, Y$ gauge bosons with $M_{V} \lesssim 10^{16} \mathrm{GeV}$ may induce observably large $D=6$ proton decay $\left(p \rightarrow \pi^{0} e^{+}\right)$. However, within the above range, the $D=6$ proton decay constraint can always be avoided by lowering $M_{\Sigma}$, which does not lead to any phenomenologicallty dangerous processes. For completeness we also report the range of the unified coupling under the assumption $M_{V}=M_{\Sigma}$. We found in the region of the plots:

$$
\begin{aligned}
\text { Vanilla SUSY : } & 24.7<\alpha^{-1}(\Lambda)<31.8 \\
\text { UGM : } & 25.0<\alpha^{-1}(\Lambda)<33.0 \\
\text { Wino DM : } & 25.3<\alpha^{-1}(\Lambda)<29.5
\end{aligned}
$$

As can be seen, $\alpha^{-1}(\Lambda)$ is always in a perturbative regime within the region of interest.

The "Vanilla" SUSY scenario, shown in the left panel of figure 1, assumes a low energy gaugino mass ratio $M_{3} / M_{2}=3$. This ratio typically arises in the scenarios where the gaugino masses are unified around the GUT scale, e.g. in the Constrained MSSM (CMSSM) or in the Gauge Mediated SUSY Breaking (GMSB) scenario. For simplicity we also assume all sfermion masses are degenerate at their mass scale, $m_{\tilde{f}}$, and $M_{3}=m_{A}=m_{\tilde{f}}$. We take $\tan \beta=2$ for all three scenarios in figure 1. For larger values of $\tan \beta$, the proton decay constraint is more constraining since the $D=5$ proton decay rate grows with some positive powers of $\tan \beta .^{5}$ In the left plot, we see that in the Vanilla SUSY scenario the proton decay constraint requires $M_{3}>500 \mathrm{TeV}$ for $\mu \sim 1 \mathrm{TeV}$ and $M_{3}>20 \mathrm{TeV}$ for $\mu \sim 10^{3} \mathrm{TeV}$.

The Universal Gaugino Mass (UGM) scenario shown in the central panel of figure 1, assumes the low energy gaugino mass ratio $M_{2} / M_{3}=1$. Such a gaugino mass ratio may arise in the gaugino focus point scenario [29-31]. For other parameters we take $M_{3}=\mu$ and $m_{A}=m_{\tilde{f}}$ as an example. We see that light SUSY spectra are allowed by the proton decay limit apart from the sfermion masses, which have $m_{\tilde{f}}>30 \mathrm{TeV}$.

The "Wino DM" SUSY scenario shown in the right panel of figure 1 has the dark matter abundance dominated by the Wino. It has been shown that the thermal Wino abundance with $M_{\widetilde{W}} \sim 3 \mathrm{TeV}$ can account for the observed energy density of the DM in the present Universe. Since the Wino becomes the lightest gaugino in the Anomaly Mediated SUSY

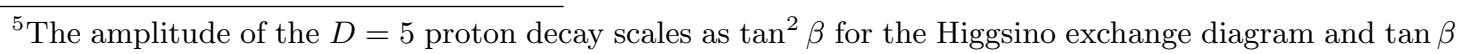
for the Wino exchange diagram. In the most region of our numerical scan, the contribution from Wino exchange diagram dominates the decay rate.
} 


\begin{tabular}{|c|c|c|c|c|c|c|}
\hline Field & $H$ & $\bar{H}$ & $\Theta$ & $\bar{\Theta}$ & $\Sigma$ & $X$ \\
\hline rep. & $\mathbf{5}$ & $\overline{\mathbf{5}}$ & $\mathbf{5 0}$ & $\overline{\mathbf{5 0}}$ & $\mathbf{7 5}$ & $\mathbf{1}$ \\
\hline $\mathrm{U}(1)_{X}$ & -2 & 1 & 2 & -1 & 0 & -1 \\
\hline
\end{tabular}

Table 2. The field content in the Higgs sector.

Breaking (AMSB) scenario, we assume the low energy gaugino mass relation $\left|M_{3} / M_{2}\right|=7$ predicted by the AMSB. For simplicity, we further assume a universal sfermion mass and $m_{A}=m_{\tilde{f}}$. In the plot, the shaded grey region is disfavoured since the sfermions are lighter than the Wino. Below the horizontal grey line, $|\mu|<M_{2}$ but we take this region into our consideration since the thermal Higgsino may account for the DM relic density in this region. We see in this plot that the current proton decay limit demands the sfermions to be heavier than $200 \mathrm{TeV}$ for $\mu \sim 1 \mathrm{TeV}$ and heavier than $40 \mathrm{TeV}$ for $\mu \sim 10^{3} \mathrm{TeV}$. This means that the split SUSY scenarios with both Wino and Higgsino DM are consistent with the minimal SU(5) model. In particular, many such scenarios predict loop suppressed gaugino masses compared to the sfermion mass, $m_{\lambda} / m_{\tilde{f}} \sim 1 / 100$. Thus Wino or Higgsino $\mathrm{DM}$ models in minimal $\mathrm{SU}(5)$ predict a proton decay lifetime in the region that may be discovered by the next generation experiments.

\section{Missing partner SU(5) models}

\subsection{Hagiwara-Yamada model}

We now study a model presented in ref. [32]. The field content in the Higgs sector is given in table 2. The superpotential of the Higgs sector is given by

$$
W_{H}=W_{1}+W_{2} \text {. }
$$

The first term is the superpotential containing only $\Sigma$ :

$$
W_{1}=M_{\Sigma} \Sigma^{2}-\frac{1}{3} \lambda_{75} \Sigma^{3}
$$

which let $\Sigma$ develop a VEV that breaks SU(5) into $G_{\mathrm{SM}}$. We have

$$
\begin{aligned}
\langle\Sigma\rangle_{[\gamma \delta]}^{[\alpha \beta]} & =\frac{1}{2}\left\{\delta_{\gamma}^{\alpha} \delta_{\delta}^{\beta}-\delta_{\delta}^{\alpha} \delta_{\gamma}^{\beta}\right\} V_{\Sigma}, \\
\langle\Sigma\rangle_{[c d]}^{[a b]} & =\frac{3}{2}\left\{\delta_{c}^{a} \delta_{d}^{b}-\delta_{d}^{a} \delta_{c}^{b}\right\} V_{\Sigma}, \\
\langle\Sigma\rangle_{[b \beta]}^{[a \alpha]} & =-\frac{1}{2}\left\{\delta_{b}^{a} \delta_{\beta}^{\alpha}\right\} V_{\Sigma},
\end{aligned}
$$

with

$$
V_{\Sigma}=\frac{3}{2} \frac{M_{\Sigma}}{\lambda_{75}}
$$

where $\alpha, \beta, \ldots$ are the $\mathrm{SU}(3)$ indices and $a, b, \ldots$ are for $\mathrm{SU}(2)$. This provides different masses for different components of $\Sigma$ and splits the $\mathbf{7 5}$ dimensional multiplet. 


\begin{tabular}{|c|c|c|c|}
\hline$\left(b_{1}, b_{2}, b_{3}\right)$ & mass & $(\mathrm{U}(1) \times \mathrm{SU}(2) \times \mathrm{SU}(3))$ & $\mathrm{SU}(5)$ \\
\hline$(-10,-6,-4)$ & $M_{V}$ & $\left(-\frac{5}{6}, \mathbf{2}, \mathbf{3}\right),\left(\frac{5}{6}, \mathbf{2}, \overline{\mathbf{3}}\right)$ & \\
\hline$\left(\frac{2}{5}, 0,1\right)$ & $M_{H_{C 1}}$ & $\left(-\frac{1}{3}, \mathbf{1}, \mathbf{3}\right),\left(\frac{1}{3}, \mathbf{1}, \overline{\mathbf{3}}\right)$ & \\
\hline$\left(\frac{2}{5}, 0,1\right)$ & $M_{H_{C 2}}$ & $\left(-\frac{1}{3}, \mathbf{1}, \mathbf{3}\right),\left(\frac{1}{3}, \mathbf{1}, \overline{\mathbf{3}}\right)$ & \\
\hline$(0,16,9)$ & $M_{\Sigma}$ & $(0, \mathbf{3}, \mathbf{8})$ & \\
\hline$(10,0,1)$ & $\frac{4}{5} M_{\Sigma}$ & $\left(\frac{5}{3}, \mathbf{1}, \mathbf{3}\right),\left(-\frac{5}{3}, \mathbf{1}, \overline{\mathbf{3}}\right)$ & \\
\hline$(10,6,10)$ & $\frac{2}{5} M_{\Sigma}$ & $\left(\frac{5}{6}, \mathbf{2}, \mathbf{6}\right),\left(-\frac{5}{6}, \mathbf{2}, \overline{\mathbf{6}}\right)$ & \\
\hline$(0,0,0)$ & $\frac{2}{5} M_{\Sigma}$ & $(0, \mathbf{1}, \mathbf{1})$ & \\
\hline$(0,0,3)$ & $\frac{1}{5} M_{\Sigma}$ & $(0, \mathbf{1}, \mathbf{8})$ & \\
\hline$\left(\frac{175}{5}, 35,34\right)$ & $M_{\Theta}$ & & $\{\mathbf{5 0}, \overline{\mathbf{5 0}}\}-\left\{\left(-\frac{1}{3}, \mathbf{1}, \mathbf{3}\right),\left(\frac{1}{3}, \mathbf{1}, \overline{\mathbf{3}}\right)\right\}$ \\
\hline
\end{tabular}

Table 3. The GUT mass spectrum and the contribution to the $\beta$-function coefficients.

The second term of the Higgs superpotential eq. (4.1) is given by

$$
W_{2}=g_{H} H \Sigma \Theta+g_{\bar{H}} \bar{H} \Sigma \bar{\Theta}+g_{X} \Theta \bar{\Theta} X .
$$

Since $\mathbf{5 0}$ does not contain a colour singlet SU(2) doublet, the second and third terms cannot give the mass to the MSSM Higgs multiplets. On the other hand, the colour triplet Higgses get masses from the two VEVs, $\langle\Sigma\rangle$ and $\langle X\rangle$, given by

$$
\hat{m}_{H_{C}}=\left(\begin{array}{cc}
0 & 4 \sqrt{3} g_{H} V_{\Sigma} \\
4 \sqrt{3} g_{\bar{H}} V_{\Sigma} & M_{\Theta}
\end{array}\right),
$$

with

$$
M_{\Theta}=g_{X}\langle X\rangle .
$$

By diagonalising this matrix one finds the two mass eigenvalues

$$
M_{H_{C 1(2)}}=\frac{M_{\Theta}}{2} \mp \frac{1}{2} \sqrt{M_{\Theta}^{2}+192 g_{H} g_{\bar{H}} V_{\Sigma}^{2}} .
$$

The GUT mass spectrum and the contribution to the $\beta$-function coefficients are given in table 3. Substituting these values into eq. (2.13) we find

$$
\begin{aligned}
\Omega_{G} & =0.729110^{-4} \cdot M_{H_{C}}^{\frac{36}{19}}\left(M_{V}^{2} M_{\Sigma}\right)^{-\frac{6}{19}} M_{\Theta}^{-\frac{18}{19}}, \\
T_{G} & =0.1683 \cdot M_{H_{C}}^{\frac{8}{19}}\left(M_{V}^{2} M_{\Sigma}\right)^{\frac{5}{19}} M_{\Theta}^{-\frac{4}{19}}, \\
C_{G} & =-46.972-30.842 \ln \frac{\Lambda}{M_{\Theta}}-20.947 \ln \frac{\Lambda}{M_{\Sigma}}-8.316 \ln \frac{\Lambda}{M_{H_{C}}}+8.1053 \ln \frac{\Lambda}{M_{V}},
\end{aligned}
$$

with

$$
M_{H_{C}}=\sqrt{M_{H_{C 1}} M_{H_{C 2}}}=48 g_{H} g_{\bar{H}} V_{\Sigma} .
$$

The numerical factors in eqs. (4.9) and (4.10) and the first term of eq. (4.11) come from the fractional numbers appearing in the masses of $\Sigma$ components in table 3 . Note that all terms 
in $C_{G}$, except for the last one, are large and negative. This drives the gauge couplings into a non-perturbative regime much before the unification scale, as shown below.

The condition for the gauge coupling unification eq. (2.7) can be recast into

$$
\begin{aligned}
\frac{M_{H_{C}}^{2}}{M_{\Theta}} & =5.4783110^{5} \cdot M_{G}^{*} \Omega_{S}\left(\frac{T_{S}}{M_{S}^{*}}\right)^{\frac{5}{6}}, \\
\left(M_{V}^{2} M_{\Sigma}\right)^{\frac{1}{3}} & =0.71554 \cdot M_{G}^{*} \Omega_{S}\left(\frac{T_{S}}{M_{S}^{*}}\right)^{-\frac{2}{9}} .
\end{aligned}
$$

It is evident from eq. (4.13) that for reasonable SUSY spectra $M_{\Theta}$ has to be much smaller than $M_{H_{C}}$. However, such configurations of the GUT masses are incompatible with perturbative gauge couplings unification. As can be seen in eq. (2.8), the contribution from the GUT threshold to the inverse of unified gauge coupling, $\alpha^{-1}(\Lambda)$, is given by $C_{G} /(2 \pi)$, which can be written by

$$
\begin{aligned}
\frac{C_{G}}{2 \pi} & =-7.476-4.909 \ln \frac{M_{H_{C}}^{2}}{M_{\Theta}}-11.14 \ln \frac{\Lambda}{M_{H_{C}}}-3.334 \ln \frac{\Lambda}{M_{\Sigma}}+1.290 \ln \frac{\Lambda}{M_{V}} \\
& =-72.34+4.909 \ln \frac{\Lambda}{S}-11.14 \ln \frac{\Lambda}{M_{H_{C}}}-3.334 \ln \frac{\Lambda}{M_{\Sigma}}+1.290 \ln \frac{\Lambda}{M_{V}},
\end{aligned}
$$

with $S=M_{G}^{*} \Omega_{S}\left(T_{S} / M_{S}^{*}\right)^{\frac{5}{6}}$. Here we used eq. (4.13) in the second equality. One can see that the first term is negative and much larger in magnitude than the constant term $\alpha_{G}^{*-1}=25.5$ in eq. (2.8). Since $M_{V}$ cannot be taken much smaller than $10^{16} \mathrm{GeV}$ to satisfy the $D=6$ proton decay constraint, the last term of eq. (4.15) cannot be large. Therefore, we conclude that it is not possible in this model to achieve the perturbative gauge coupling unification in phenomenologically allowed parameter region, unless the SUSY contribution $\frac{C_{S}}{2 \pi}$ is positive and very large and/or $S \ll \Lambda$. We do not consider such a possibility since it would require extreme mass hierarchies in the MSSM spectrum.

\subsection{Hisano-Moroi-Tobe-Yanagida model}

A solution to the problem found in the Hagiwara-Yamada model was proposed by Hisano, Moroi, Tobe and Yanagida [4]. The main idea is to implement a structure to suppress the $D=5$ proton decay and to make the $\Theta$ fields very heavy so that the second term in eq. (4.11) is made small. In their model new fields, distinguished with primes, are introduced in the $H, \bar{H}, \Theta, \bar{\Theta}$ sectors with appropriate $\mathrm{U}(1)_{X}$ charges: $H^{\prime}(2), \bar{H}^{\prime}(-1)$, $\Theta^{\prime}(1), \bar{\Theta}^{\prime}(-2)$. Notice that now $\Theta$ fields can have tree-level mass terms with primed fields. The superpotential for the primed fields can be written as

$$
W^{\prime}=g_{\bar{H}}^{\prime} \bar{H}^{\prime} \Sigma \Theta^{\prime}+g_{H}^{\prime} H^{\prime} \Sigma \bar{\Theta}^{\prime}+M_{1} \bar{\Theta} \Theta^{\prime}+M_{2} \bar{\Theta}^{\prime} \Theta+g_{X} \bar{H}^{\prime} H^{\prime} X .
$$

We assume $M_{1}, M_{2} \gg\langle\Sigma\rangle,\langle X\rangle$ so that the mass splitting within the $\Theta$ multiplets can be neglected, and the effective superpotential after integrating out the $\Theta$ fields can be used at the energy scale of the coupling unification. Substituting the VEVs of $\Sigma$ and integrating out the $\Theta$ fields, one finds the effective colour triplet Higgs mass terms

$$
M_{H_{C_{1}}} \bar{H}_{C} \bar{H}_{C}^{\prime}+M_{H_{C_{2}}} \bar{H}_{C}^{\prime} \bar{H}_{C},
$$


with $M_{H_{C_{1}}} \simeq 48 g_{H} g_{\bar{H}}^{\prime} V_{\Sigma}^{2} / M$ and $M_{H_{C_{2}}} \simeq 48 g_{H}^{\prime} g_{\bar{H}} V_{\Sigma}^{2} / M$, where $M_{1}=M_{2}=M$ is taken for simplicity. The last term of $W^{\prime}$ introduces the mass to the doublet and triplet fields in $H^{\prime}, \bar{H}^{\prime}$

$$
M_{f}\left(\bar{H}_{f}^{\prime} H_{f}^{\prime}+\bar{H}_{C}^{\prime} H_{C}^{\prime}\right)
$$

with $M_{H_{f}} \equiv g_{X}\langle X\rangle$.

Due to the $\mathrm{U}(1)_{X}$ symmetry, $H^{\prime}$ and $\bar{H}^{\prime}$ cannot have Yukawa interactions with matter fields. Since there is no direct mass term between $H_{C}$ and $\bar{H}_{C}$, the propagators of $H_{C}$ and $\bar{H}_{C}$ cannot be connected in the $D=5$ proton decay operator by themselves. The leading contribution comes via the mixing between the primed and unprimed triplet Higgs fields together with the the direct mass term $M_{H_{f}} \bar{H}_{C}^{\prime} H_{C}^{\prime}$. Therefore, the $D=5$ proton decay operator receives an extra suppression $M_{H_{f}} / M_{H_{C}}$ compared to the previous model with $M_{H_{C}}=\sqrt{M_{H_{C 1}} M_{H_{C 2}}}$.

The change to our formulae for $\Omega_{G}, T_{G}, C_{G}$ from the previous model is as follows. Now the entire $\Theta, \bar{\Theta}, \Theta^{\prime}, \bar{\Theta}^{\prime}$ multiplets (including triplet components) are decoupled and absent at the scale of the coupling unification. Instead, new pairs of triplet and doublet (coming from $H^{\prime}$ and $\bar{H}^{\prime}$ ) are present and contributing to the GUT threshold correction. For $\Omega_{G}$ and $T_{G}$, we found the same expressions as eqs. (4.9) and (4.10) by replacing $M_{\Theta} \rightarrow M_{H_{f}}$ and $M_{H_{C}}=\sqrt{M_{H_{C 1}} M_{H_{C 2}}}=48 \sqrt{g_{H} g_{\bar{H}} g_{H}^{\prime} g_{\bar{H}}^{\prime}} V_{\Sigma}^{2} / M$. Because of this, the conditions of the gauge coupling unification, eqs. (4.13) and (4.14), are also unchanged up to the replacement $M_{\Theta} \rightarrow M_{H_{f}}$ in the former, which reads

$$
\frac{M_{H_{C}}^{2}}{M_{H_{f}}}=5.4783110^{5} \cdot M_{G}^{*} \Omega_{S}\left(\frac{T_{S}}{M_{S}^{*}}\right)^{\frac{5}{6}} .
$$

For $\frac{C_{G}}{2 \pi}$, we have

$$
\begin{aligned}
\frac{C_{G}}{2 \pi} & =-7.476+0.502 \ln \frac{\Lambda}{M_{H_{f}}}-1.323 \ln \frac{\Lambda}{M_{H_{C}}}-3.333 \ln \frac{\Lambda}{M_{\Sigma}}+1.290 \ln \frac{\Lambda}{M_{V}}, \\
& =-0.834-0.502 \ln \frac{\Lambda}{S}-0.318 \ln \frac{\Lambda}{M_{H_{C}}}-3.333 \ln \frac{\Lambda}{M_{\Sigma}}+1.290 \ln \frac{\Lambda}{M_{V}},
\end{aligned}
$$

where we have used eq. (4.19) in the second equality. Contrary to eq. (4.15), we see that the GUT contribution to the unified coupling is much smaller than the leading constant term $\alpha_{G}^{*}=25.5$. The perturbative coupling unification can therefore be easily achieved with ordinary SUSY spectra. The $D=5$ proton decay depends on the "effective triplet" mass given by the ratio $M_{H_{C}}^{2} / M_{H_{f}}$ and, as shown in figure 2, is far beyond the experimental reach (in a foreseeable future) for a realistic SUSY spectrum. The $D=6$ dependent decay channel depends on the $\Sigma$ mass, which is a free parameter of the model. We report the ranges of the $\left(M_{V}^{2} M_{\Sigma}\right)^{\frac{1}{3}}$ and $\alpha^{-1}(\Lambda)$ (computed assuming $M_{V}=M_{\Sigma}$ ) obtained in the scan of the $\left(m_{\tilde{f}}, \mu\right)$ plane in figure 2 :

$$
\begin{aligned}
3.21 \cdot 10^{15} \mathrm{GeV} & <\left(M_{V}^{2} M_{\Sigma}\right)^{\frac{1}{3}}<1.49 \cdot 10^{16} \mathrm{GeV}, \\
39.6 & <\alpha^{-1}(\Lambda)<47.9,
\end{aligned}
$$

where the nominal value of $\alpha_{s}\left(m_{Z}\right)$ was used. 


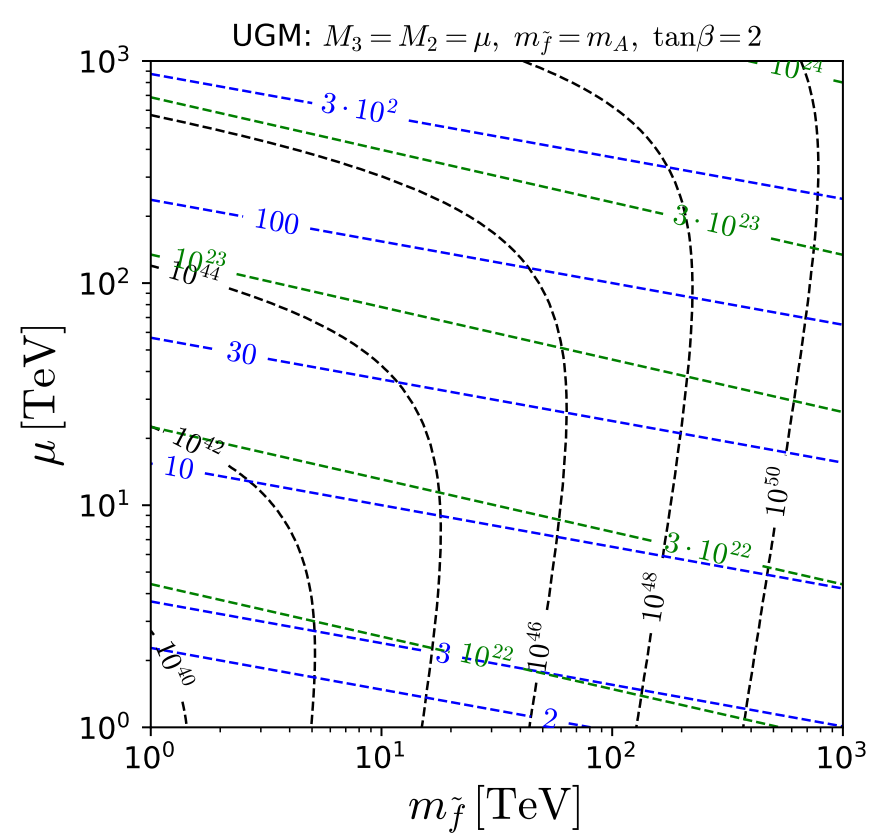

Figure 2. The low energy SUSY parameter space $\left(m_{\tilde{f}}\right.$ versus $\mu$ plane) for the UGM scenario with $M_{3}=M_{2}=\mu, m_{A}=m_{\tilde{f}}$ and $\tan \beta=2$. The dashed black, green and blue contours represent the values of $\tau\left(p \rightarrow K^{+} \bar{\nu}\right) / \mathrm{yrs}, M_{H_{C}} / \mathrm{GeV}$ and $T_{S} / \mathrm{TeV}$, respectively, calculated in the missing partner $\mathrm{SU}(5)$ model in ref. [4].

\section{$5 \quad$ SU(5) orbifold SUSY GUT}

In this section we study the implication of gauge coupling unification in the $5 \mathrm{~d}$ SU(5) orbifold SUSY model presented in ref. [5]. In this model, space-time is assumed to be the cross product of ordinary $4 \mathrm{~d}$ Minkowski spacetime with a $S^{1} /\left(Z_{2} \times Z_{2}^{\prime}\right)$ orbifold. This orbifold has two fixed points $O$ and $O^{\prime}$ and one can assign to the fields two independent $Z_{2}$ charges, $P$ and $P^{\prime}$, corresponding to the reflection symmetries, $y \rightarrow-y$, of the $S^{1}$ coordinate $y$, centred around $O$ and $O^{\prime}$, respectively. Since the spinor representation in the $5 \mathrm{~d}$ spacetime has 8 real components, supersymmetry in the bulk is doubled compared to those in the $4 \mathrm{~d}$ spacetime. As a consequence, the $5 \mathrm{~d}$ vector multiplet $\mathcal{V}(V, \Sigma)$ contains a 4 d vector multiplet $V$ and a 4 d chiral multiplet $\Sigma$ in the adjoint representation. The Higgs fields are introduced in the bulk and embedded in the hypermultiplets, $\mathcal{H}\left(H(\mathbf{5}), H^{c}(\overline{\mathbf{5}})\right)$ and $\overline{\mathcal{H}}\left(\bar{H}(\overline{\mathbf{5}}), \bar{H}^{c}(\mathbf{5})\right)$, containing two $4 \mathrm{~d}$ chiral multiplets in a vector like manner; $\{H$, $\left.H^{c}\right\} \supset\left\{\left(H_{F}, H_{C}\right),\left(H_{F}^{c}, H_{C}^{c}\right)\right\}$ and $\left\{\bar{H}, \bar{H}^{c}\right\} \supset\left\{\left(H_{\bar{F}}, H_{\bar{C}}\right),\left(H_{\bar{F}}^{c}, H_{\bar{C}}^{c}\right)\right\}$, respectively, where the subscript $F(C)$ represents the doublet (triplet) component. To break the SU(5) into $G_{\mathrm{SM}}$, the orbifold parities are assigned so that only the $4 \mathrm{~d}$ gauge multiplets corresponding to the $G_{\mathrm{SM}}$ generators have zero modes. More specifically, $V^{a}(+,+)$ and $V^{\hat{a}}(+,-)$ are taken for $\left(P, P^{\prime}\right)$, where $a(\hat{a})$ corresponds to the unbroken (broken) generators. This parity assignment implies that the $\mathrm{SU}(5)$ is broken at a 3-brane at $O^{\prime}$ but unbroken at the other brane at $O$. In order to preserve the success of the charge quantization and assignment in the matter sector in the $4 \mathrm{~d}$ GUTs, the matter fields $F_{i}=\left(D^{c}, L\right)_{i}$ and $T_{i}=\left(Q, U^{c}, E^{c}\right)_{i}$, $(i=1,2,3)$, are placed at the $\mathrm{SU}(5)$ symmetric 3 -brane at $O$. 


\begin{tabular}{|c|c|c|c|c|}
\hline$K K$ mode & mass & $\left(P, P^{\prime}\right)$ & 4 d fields & $\sum\left(b_{1}, b_{2}, b_{3}\right)$ \\
\hline zero & 0 & $(+,+)$ & $V^{a}, H_{F}, H_{\bar{F}}$ & \\
\hline \multirow{2}{*}{ even } & \multirow{2}{*}{$(2 n+2) / R$} & $(+,+)$ & $V^{a}, H_{F}, H_{\bar{F}}$ & \multirow{2}{*}{$\left(\frac{6}{5},-2,-6\right)$} \\
\cline { 3 - 5 } & & $(-,-)$ & $\Sigma^{a}, H_{F}^{c}, H_{\bar{F}}^{c}$ & \\
\hline \multirow{2}{*}{ odd } & \multirow{2}{*}{$(2 n+1) / R$} & $(+,-)$ & $V^{\hat{a}}, H_{C}, H_{\bar{C}}$ & \multirow{2}{*}{$\left(-\frac{46}{5},-6,-2\right)$} \\
\cline { 3 - 4 } & & $(-,+)$ & $\Sigma^{\hat{a}}, H_{C}^{c}, H_{\bar{C}}^{c}$ & \\
\hline
\end{tabular}

Table 4. The orbifold parity assignment and contributions to the $\beta$-function coefficients from the bulk fields. $R$ is the compactification radius.

In table 4 we show the complete orbifold parity assignment for the bulk fields, in addition to the total contribution to $\beta$-function coefficients from the even and odd $K K$ excitations. In the table, we separate out the zero mode, since they are included in the MSSM. With this charge assignment, the doublet-triplet splitting problem is elegantly solved because only two doublet Higgs fields can have zero modes.

The structure to suppress the $D=5$ proton decay discussed in subsection 4.2 is automatically implemented in this model. This is because the $K K$-mass is generated only amongst the components residing in the same $5 \mathrm{~d}$ multiplet; e.g. $W \supset \frac{(2 n+1)}{R}\left[H_{C}^{(2 n+1)} H_{C}^{c(2 n+1)}+H_{\bar{C}}^{(2 n+1)} H_{\bar{C}}^{c(2 n+1)}\right]$, whereas there are no direct mass terms connecting two fields from different $5 \mathrm{~d}$ multiplets, such as $H_{C}^{(2 n+1)} H_{\bar{C}}^{(2 n+1)}$ and $H_{C}^{c(2 n+1)} H_{\bar{C}}^{c(2 n+1)}$. This can be also understood in terms of a $\mathrm{U}(1)_{R}$ symmetry of this model with the following charge assignment; $H(0), H^{c}(2), \bar{H}(0), \bar{H}^{c}(2), F_{i}(1), T_{i}(1)$. One can see that due to this $\mathrm{U}(1)_{R}$ symmetry only $H(0) \supset H_{C}$ and $\bar{H}(0) \supset H_{\bar{C}}$ can have Yukawa couplings to the matter fields. Since $H_{C}$ and $H_{\bar{C}}$ do not couple via a mass term, the $D=5$ proton decay operator is not generated. In other words $D=5$ proton decay is forbidden by the $\mathrm{U}(1)_{R}$ symmetry.

In calculating the threshold corrections we follow ref. [5] and assume that the $5 \mathrm{~d}$ theory is cut-off at a scale $\Lambda$ where the field theory is presumably incorporated in some more fundamental theory. There are two sources of the GUT threshold corrections in this model. One is from mass splitting among the $K K$-even and -odd mode multiplets, as shown in table 4 . This part can be treated with $T_{G}, \Omega_{G}$ and $C_{G}$ by the formula (2.13). The other source is from the brane kinetic term at $O^{\prime}$. This is because the $4 \mathrm{~d} \operatorname{SU}(5)$ gauge symmetry is explicitly broken into $G_{\mathrm{SM}}$ at the $O^{\prime}$ brane because there is no $4 \mathrm{~d}$ gauge fields corresponding to the broken generators at $O^{\prime}$. This means that one can introduce independent kinetic terms for the three MSSM gauge fields with different gauge couplings. However, in general the bulk contribution to the $4 \mathrm{~d}$ gauge coupling always dominates due to the spread of the wave function and the contribution from the brane kinetic term is suppressed by the volume factor $2 \pi R \Lambda$ where $R$ is the radius of $S^{1}{ }^{6}$ In the following analysis, we neglect the contribution from the brane kinetic terms. ${ }^{7}$

\footnotetext{
${ }^{6}$ For example, it has been estimated in ref. [5] that the contribution to the weak mixing angle from the brane kinetic terms is much less than $1 \%$ for $r \equiv R \Lambda=4$.

${ }^{7}$ Once the brane couplings are specified, their effect can be included into the analysis by adding appropriate constants in the set of eq. (2.1) and in the first set of eq. (2.4).
} 
Coming back to the mass splitting among the $K K$ GUT multiplets due to the GUT breaking parity assignment. Neglecting a finite correction from the brane kinetic term, the following picture is expected [5]. Evolving the gauge couplings from low energy to high energy, they approach each other in the MSSM. After passing the compactification scale, $M_{c}=1 / R, K K$-modes appear and the running changes. In the $\mu>M_{c}$ regime, the running is slower but the gauge couplings continue to approach each other. The three gauge couplings approximately meet at the cut-off scale, $\Lambda$, where the $5 \mathrm{~d}$ theory may be incorporated into a more fundamental theory. Namely, the unification scale $\Lambda$ in eq. (2.1) serves also as the cut-off scale in this model.

As before the gauge coupling unification condition can be expressed in terms of $T_{G}, \Omega_{G}$ and $C_{G}$. From eq. (2.13) and table 4 , one can see that the contributions to $\ln \Omega_{G}, \ln \left(T_{G} / \Lambda\right)$ and $C_{G}$ from all the odd $K K$ excitations with level $(2 k+1)$ takes the form

$$
c_{o} \ln \frac{2 k+1}{r},
$$

where $r=\Lambda R$. Those from the even excitations with level $(2 k+2)$ are given by

$$
c_{e} \ln \frac{2 k+2}{r}
$$

where $c_{o}=-c_{e}=\frac{24}{19}$ for $\ln \Omega_{G}, c_{o}=-c_{e}=\frac{18}{19}$ for $\ln \left(T_{G} / \Lambda\right)$ and $c_{o}=\frac{4}{19}, c_{e}=-\frac{156}{19}$ for $C_{G}$. The index $k$ runs from 0 to $k_{\max }^{o / e}$ with $2 k_{\max }^{o}+1 \leq r$ for odd and $2 k_{\max }^{e}+2 \leq r$ for even excitations. Summing over $k$ up to $k_{\max }^{o / d}$, we arrive at the expression

$$
\begin{aligned}
\Omega_{G} & =\left[\frac{\prod_{k}^{k_{\max }^{o}}(2 k+1)}{\prod_{k}^{k_{\max }^{e}}(2 k+2)}\left(\frac{1}{r}\right)^{k_{\max }^{o}-k_{\max }^{e}}\right]^{\frac{24}{19}}, \\
\frac{T_{G}}{\Lambda} & =\left[\frac{\prod_{k}^{k_{\max }^{o}}(2 k+1)}{\prod_{k}^{k_{\max }^{e}}(2 k+2)}\left(\frac{1}{r}\right)^{k_{\max }^{o}-k_{\max }^{e}}\right]^{\frac{18}{19}}, \\
C_{G} & =\frac{4}{19} \ln \left[\prod_{k=0}^{k_{\max }^{o}} \frac{2 k+1}{r}\right]-\frac{156}{19} \ln \left[\prod_{k=0}^{k_{\max }^{e}} \frac{2 k+2}{r}\right] .
\end{aligned}
$$

Unlike in the 4d GUT models studied in previous sections, $T_{G}$ and $\Omega_{G}$ are dependent on $\Lambda$ (explicitly or implicitly through $r$. See appendix A.3 for the reason why this is the case.) We note that $\Omega_{G}$ and $T_{G} / \Lambda$ are simply related by

$$
\frac{T_{G}}{\Lambda}=\Omega_{G}^{\frac{3}{4}} .
$$

As examples, the explicit forms of $\Omega_{G}$ and $T_{G} / \Lambda$ for the first few ranges of $r$ are given in table 5. It is worth stressing that in this model $\Omega_{G}, T_{G} / \Lambda$ and $C_{G}$ are constants for a given $r$. The first equation, $T_{S}=M_{S}^{*} \Omega_{G}$, of the unification condition eq. (2.7) therefore places a non-trivial constraint amongst the low energy SUSY masses as

$$
T_{S}=\left[M_{3}^{-28} M_{2}^{32} \mu^{12} m_{A}^{3} X_{T}\right]^{\frac{1}{19}}=M_{S}^{*} \Omega_{G},
$$




\begin{tabular}{|c|c|c|c|c|c|}
\hline & $1<r \leq 2$ & $2<r \leq 3$ & $3<r \leq 4$ & $4<r \leq 5$ & $\cdots$ \\
\hline$\Omega_{G}$ & {$\left[\frac{1}{r}\right]^{\frac{24}{19}}$} & {$\left[\frac{1}{2}\right]^{\frac{24}{19}}$} & {$\left[\frac{1 \cdot 3}{2} \frac{1}{r}\right]^{\frac{24}{19}}$} & {$\left[\frac{1 \cdot 3}{2 \cdot 4}\right]^{\frac{24}{19}}$} & $\cdots$ \\
$T_{G} / \Lambda$ & {$\left[\frac{1}{r}\right]^{\frac{18}{19}}$} & {$\left[\frac{1}{2}\right]^{\frac{18}{19}}$} & {$\left[\frac{1 \cdot 3}{2} \frac{1}{r}\right]^{\frac{18}{19}}$} & {$\left[\frac{1 \cdot 3}{2 \cdot 4}\right]^{\frac{18}{19}}$} & $\cdots$ \\
\hline
\end{tabular}

Table 5. The explicit forms for $\Omega_{G}$ and $T_{G} / \Lambda$ for the first few ranges of $r=R \Lambda$.

where eq. (2.11) was used. As examples, $T_{S}=(1612,1276,1031) \mathrm{GeV}$ for $r=1.5$ and (779, $617,498) \mathrm{GeV}$ for $r=4$ for $\alpha_{s}\left(m_{Z}\right)=(0.1175,0.1183,0.1191)$.

The second equation of the unification condition is equivalent to $T_{G} / \Lambda=M_{G}^{*} \Omega_{S} / \Lambda$, where the left-hand-side is constant and can be traded with $\Omega_{G}^{\frac{3}{4}}$ using eq. (5.4). This allows for the determination of the cut-off scale as a function of SUSY masses through $\Omega_{S}$ giving

$$
\begin{aligned}
\Lambda & =M_{G}^{*} \Omega_{G}^{-\frac{3}{4}} \Omega_{S} \\
& =M_{G}^{*} \Omega_{G}^{-\frac{31}{54}} M_{s}^{* \frac{19}{108}}\left(\frac{M_{3}}{M_{2}}\right)^{\frac{19}{216}} M_{3}^{-\frac{38}{216}} X_{T}^{-\frac{1}{108}} X_{\Omega}^{\frac{1}{288}},
\end{aligned}
$$

where the second equality is obtained by computing $\Omega_{S}$ under the constraint of eq. (5.5). In this process we have eliminated the combination $\left(\mu^{4} m_{A}\right)^{\frac{1}{5}}$.

Since $\Lambda$ is related to the mass of the first $K K X, Y$ bosons by $M_{c}=\Lambda / r$, for a given $r$ we can compute the $D=6$ proton decay, $p \rightarrow \pi^{0} e^{+}$, from the low energy SUSY spectrum. We show in figure 3 the $\left(M_{3}, M_{3} / M_{2}\right)$ plane of the MSSM for $\Lambda / M_{c}=1.5$ (left) and 4 (right). For simplicity, we assume sfermion masses $\left(m_{\tilde{f}}\right)$ are universal at low energy, which assures $X_{T}=X_{\Omega}=1$. We impose eqs. (5.5) and (5.6) so that the gauge couplings unify at $\Lambda$. The former constraint allows one to determine $\left(\mu^{4} m_{A}\right)^{\frac{1}{5}}$ at each point of the plane. The dashed blue lines shows the contours of $\left(\mu^{4} m_{A}\right)^{\frac{1}{5}}$ required for the unification using the nominal value of $\alpha_{s}$. The region with $\left(\mu^{4} m_{A}\right)^{\frac{1}{5}}<100 \mathrm{GeV}$ marked by blue predicts an unacceptably light chargino or non-SM Higgs bosons because one of the $\mu$ and $m_{A}$ (or both) is less than $100 \mathrm{GeV}$. The dotted-dashed and dotted blue lines correspond to the same contour $\left(\mu^{4} m_{A}\right)^{\frac{1}{5}}=100 \mathrm{GeV}$ but obtained from upper and lower 1- $\sigma$ variations of $\alpha_{s}\left(m_{Z}\right)$, respectively.

Light gluinos are strongly constrained by the null result of SUSY searches at the LHC. We take the most conservative bound on the gluino mass [33-35] and mark the excluded region, $M_{3}<1 \mathrm{TeV}$, by grey. This limit generally applies if the spectrum is compressed, while more severe bound should be applied otherwise. When the mass difference between gluino and the lightest SUSY particle (LSP) is large, ${ }^{8}$ the gluino mass is excluded up to $\sim 2.0-2.2 \mathrm{TeV}$ by the current data [33-36].

As discussed above, the mass of the $X, Y$ gauge bosons is determined at each point of the parameter plane. We evaluate the unified coupling using eq. (2.8) and $C_{S}$ and $C_{G}$, assuming $m_{\tilde{f}}=M_{3}$ and $\mu=m_{A}$, but the dependency on the SUSY spectrum is very mild. Then, the proton decay lifetime $\tau\left(p \rightarrow \pi^{0} e^{+}\right)$can be calculated. The black dashed

\footnotetext{
${ }^{8}$ The running of the gauge coupling is independent of the Bino mass, which is a gauge singlet. The lower bound of the LSP mass is therefore unconstrained, since the LSP can be Bino-like.
} 


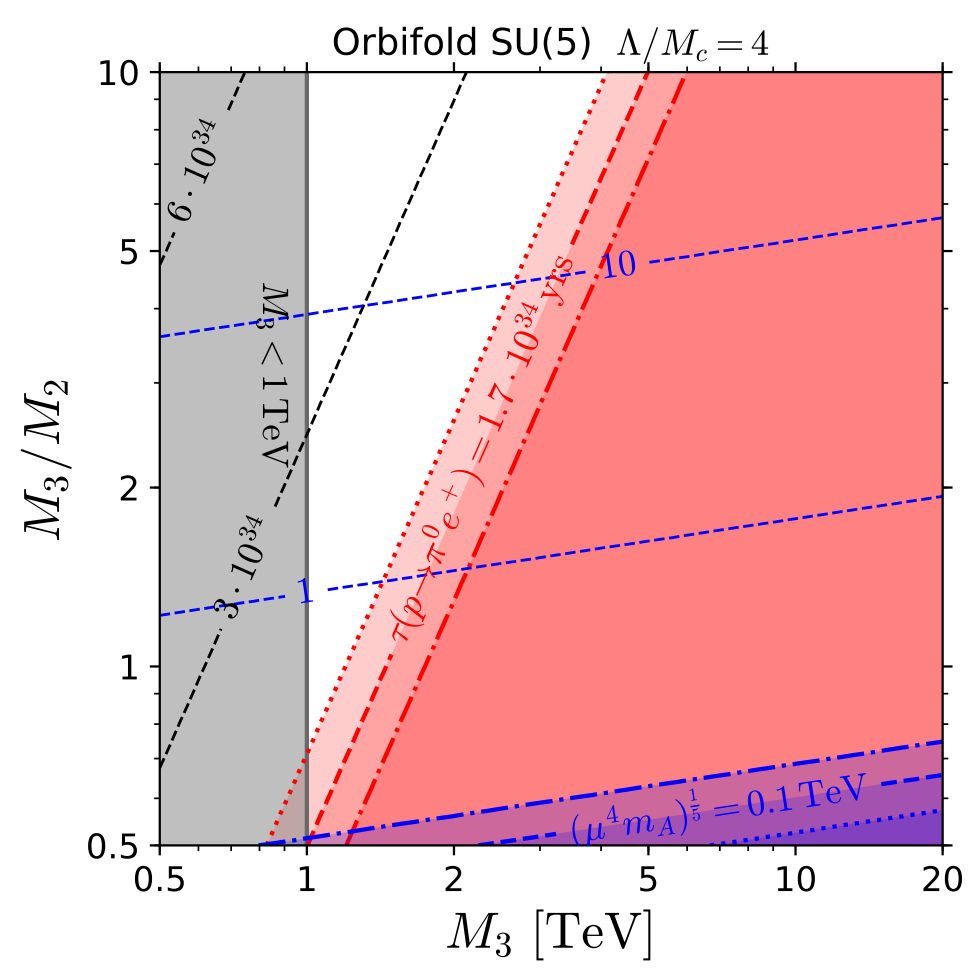

Figure 3. The $M_{3}$ versus $M_{3} / M_{2}$ plane in the orbifold SU(5) SUSY-GUT model [5] with $r=R \Lambda=$ 4. The grey shaded region shows a conservative limit on the gluino mass from the direct SUSY searches at the LHC. The blue dashed lines are the contours of $\left(\mu^{4} m_{A}\right)^{\frac{1}{5}}$ required by the gauge coupling unification. The shaded blue region is phenomenologically excluded due to the presence of too light charginos or non-SM Higgs bosons. The black dashed lines are the contours of the $\tau\left(p \rightarrow \pi^{0} e^{+}\right)$predicted due to the condition of gauge coupling unification. The shaded red region is excluded due to the present proton decay bound, $\tau\left(p \rightarrow \pi^{0} e^{+}\right)<1.7 \cdot 10^{34} \mathrm{yrs}$. The black and blue dashed lines are obtained using the nominal value of $\alpha_{s}\left(m_{Z}\right)$, whilst the dotted-dashed and dotted lines (red and blue) are obtained using the upper and lower variation of $\alpha_{s}\left(m_{Z}\right)$ corresponding to the $1-\sigma$ uncertainty.

lines show the contours of the $\tau\left(p \rightarrow \pi^{0} e^{+}\right)$using the nominal value of $\alpha_{s}$. The current proton decay limit, $\tau\left(p \rightarrow \pi^{0} e^{+}\right)<1.7 \cdot 10^{34} \mathrm{yrs}$, excludes the region shaded by red. The dotted-dashed and dotted red lines represent the contours of $\tau\left(p \rightarrow \pi^{0} e^{+}\right)<1.7 \cdot 10^{34} \mathrm{yrs}$ obtained from upper and lower 1- $\sigma$ variations of $\alpha_{s}\left(m_{Z}\right)$, respectively. The ranges of $\Lambda$ and $\alpha^{-1}(\Lambda)$ found for $r=4$ in the parameter range of the right plot in figure 3 are

$$
\begin{aligned}
1.58 \cdot 10^{16} \mathrm{GeV} & <\Lambda<4.19 \cdot 10^{16} \mathrm{GeV}, \\
26.2 & <\alpha(\Lambda)<28.4 .
\end{aligned}
$$

As can be seen, this model is constrained strongly by the LHC and the proton decay measurement. For $r>4$, the constraint is even tighter. This is because the proton lifetime is roughly proportional to $M_{c}^{4}=(\Lambda / r)^{4}$. Although $\Lambda$ is larger for larger $r$ (see eq. (5.6) and the dependence of $\Omega_{G}$ on $r$ ) this effect is very mild as compared to the suppression by the fourth power of $r$ in the expression for $M_{c}$. It also follows from eq. (5.6) that for 
fixed values of $r$ and the ratio $M_{3} / M_{2}$ proton life time is shorter for larger values of $M_{3}$ (the value of $\Lambda$ is then decreasing with increasing $M_{3}$ ). The upper bound on $M_{3}$ following from the present limit on proton lifetime is larger for smaller value of $r$ and larger value of the ratio $M_{3} / M_{2}$, as both increase the value of $\Lambda$ (see eq. (5.6)). It is clear that further improvement of the collider constraint as well as the proton lifetime limit will cover the entire parameter space of this model.

\section{Discussions and conclusions}

In this paper, we have derived analytic expressions for the condition of gauge coupling unification (eq. (2.7)) and the unified gauge coupling (eq. (2.8)) in terms of the masses of SUSY and GUT particles. The formula is generic and applicable for any GUT models in which the SM gauge group is directly unified into a simple unified gauge group at some high energy scale. The unification condition, eq. (2.7), is expressed in a form of two simultaneous equations, which are simple (and symmetric) relations between the four variables $T_{S}, \Omega_{S}$, $T_{G}$ and $\Omega_{G}$. This is advantageous because, no matter how complicated the GUT models are, the condition can be written in terms of only those four variables.

The first two variables $\left(T_{S}\right.$ and $\left.\Omega_{S}\right)$ are functions of superparticle masses and their explicit forms in the MSSM are given in eq. (3.6). The formula is derived from the RGE equation including the 2-loop effect, while the threshold correction is treated at 1-loop. Therefore, the condition is insensitive to the mass of a particle that is singlet under the SM gauge group, such as the Bino. Thus, the gauge coupling unification (GCU) condition alone cannot determine what the lightest SUSY particle is in the MSSM. Similarly, the MSSM formula eq. (3.6) is unchanged even for singlet extensions of the MSSM, such as next-to-MSSM (NMSSM). For non-singlet extensions of the MSSM, the corresponding formula can be found straightforwardly by solving the second set of linear equations in eq. (2.4) with eq. (2.2).

The remaining two variables $\left(T_{G}\right.$ and $\left.\Omega_{G}\right)$ are functions of the GUT masses. The expressions in eq. (2.13) are generic for any GUT model with gauge coupling unification. Despite their appearance, $\Omega_{G}$ and $T_{G}$ are independent of the unification scale $\Lambda$ for conventional 4d GUT models. On the other hand, they may be dependent on $\Lambda$ in GUT models in higher dimensions, and we have seen this is indeed the case in the orbifold SU(5) model in section 5. A more detailed discussion of the $\Lambda$ dependence of the condition for the GCU is given in the appendix.

The GCU condition and the unified gauge coupling are of course subject to the uncertainty in the measured value of the strong coupling constant at the weak scale, $\alpha_{s}\left(m_{Z}\right)$. In our formalism this uncertainty is conveniently treated by understanding the effect of $\alpha_{s}\left(m_{Z}\right)$ on the three constants $M_{S}^{*}, M_{G}^{*}$ and $\alpha_{G}^{*}$ appearing in the analytic formula. The dependence on the value of $\alpha_{s}\left(m_{Z}\right)$ of these constants is numerically parametrised in eq. (2.5).

In section 3, Minimal SU(5) SUSY GUT is studied using our analytical formulae. We have found that the GCU conditions are re-expressed as the formula for the coloured Higgs mass (eq. (3.7)), and that for $\left(M_{V}^{2} M_{\Sigma}\right)$ (eq. (3.8)), as functions of the superparticle masses. Using the former formula, one can predict the $D=5$ proton decay mediated by the 
superparticles and the coloured higgsinos entirely in terms of the MSSM mass spectrum. We have shown in figure 1 the $D=5$ proton decay lifetime in several slices of SUSY parameter space. The $D=5$ proton decay constraint is quite severe for the Minimal SU(5) SUSY models. However, relatively light SUSY spectrum $\left(M_{3}, M_{2}, \mu \sim \mathcal{O}(1) \mathrm{TeV}\right)$ is possible if sfermion masses are taken to be large, $m_{\tilde{f}} \gtrsim 30 \mathrm{GeV}$.

Two missing partner SU(5) models have been discussed in section 4, where the doublet triplet splitting is naturally solved. In Hagiwara-Yamada model [32], we have shown analytically that the GCU condition cannot be made consistent with the perturbativity of gauge couplings with reasonable SUSY spectra. In Hisano-Moroi-Tobe-Yanagida model [4], this problem is solved by making the $\Theta$ field very heavy. In this model, the $D=5$ proton decay is very suppressed and the predicted values are beyond the next generation nucleon decay experiments.

The 5d orbifold SU(5) SUSY GUT model [5] has also been studied in section 5. We have shown that the variables $T_{G}, \Omega_{G}$ and $C_{G}$ are effectively functions of the cut-off scale (unification scale), $\Lambda$, and $r=R \Lambda$, which determine the $K K$ spectrum. The GCU condition imposes a non-trivial constraint on the MSSM spectrum for given $r$, and the cut-off scale is also determined. In figure 3 we have shown the collider and $D=6$ proton decay constraint in the $\left(M_{3} / M_{2}\right.$ vs $\left.M_{3}\right)$ parameter plane. Since the $X, Y$ gauge boson mass (i.e. the compactification scale) is proportional to $M_{3}^{-\frac{38}{216}}$ (see eq. (5.6)) the larger the gluino mass the faster $D=6$ proton decay is predicted. Combining the gluino mass bound from the collider search, we have found a complementarity between the collider and proton decay experiments in testing this model. It has been shown that the $5 \mathrm{~d}$ orbifold $\mathrm{SU}(5)$ SUSY GUT [5] is already very severely constrained by the LHC and the $D=6$ proton decay measurement.

\section{Acknowledgments}

The work of SP, KR and KS is partially supported by the Beethoven grants DEC2016/23/G/ST2/04301. The work of SP and KR are partially supported by the Harmonia grants DEC-2015/18/M/ST2/00054. The work of KS is partially supported by the National Science Centre, Poland, under research grants 2017/26/E/ST2/00135. The work of KR is partially supported by the National Science Centre, Poland, under research grants 2015/19/D/ST2/03136.

\section{A Interpretations of threshold corrections}

In this appendix we compare two different formulations and interpretations of the 1-loop threshold correction from superheavy particles and discuss the condition of the GCU in each case. We also comment on orbifold GUT models, where the GCU may look accidental from the $4 \mathrm{~d}$ field theoretical point of view. 

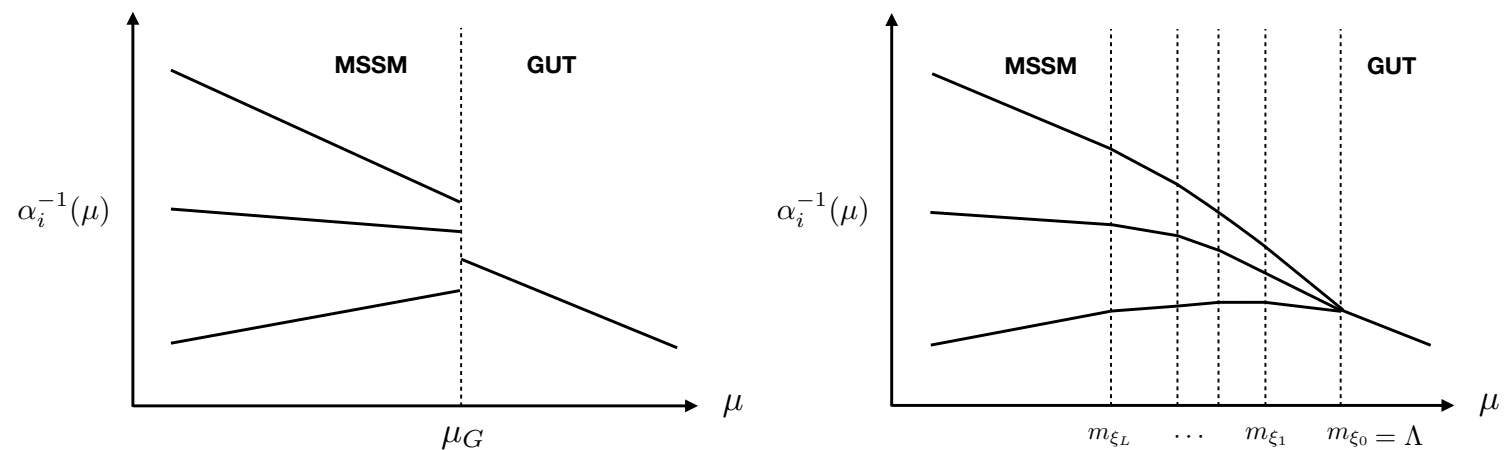

Figure 4. The RGE evolution of the gauge couplings in two different pictures: Left: a single EFT matching (from GUT to MSSM) at $\mu_{G}$. Right: sequential EFT matchings at the particle masses. Here, the unification scale, $\Lambda$, is given by the mass of the heaviest particle, $m_{\xi_{0}}$, that forms an incomplete GUT multiplet.

\section{A.1 Mass independent unified gauge coupling}

One way to find the relation between the MSSM parameters and the GUT model is to derive the MSSM as a low energy effective field theory (EFT) of the GUT model simply by integrating out all the superheavy particles and expressing the result in terms of the single, mass independent, minimal subtraction coupling constant of the GUT group [25]. The strategy is to stay away from thresholds where the scale dependence of couplings is complicated. This is done by computing the difference between the coupling at $\mu_{G}$ below the heavy masses to the scale $\mu_{2}$ well above the masses, analytically integrating out the heavy states, and avoiding the need numerically to integrate through the threshold. The result, equivalent to the analyses in $[24,26]$, may be written as the boundary condition for the running coupling constants

$$
\frac{2 \pi}{\alpha\left(\mu_{G}\right)}=\frac{2 \pi}{\alpha_{i}\left(\mu_{G}\right)}+r_{i}\left(\mu_{G}\right)
$$

where $\alpha\left(\mu_{G}\right)$ on the left-hand-side is the unified gauge coupling of the GUT model and $\alpha_{i}\left(\mu_{G}\right)$ on the right-hand-side are the MSSM gauge couplings evaluated at $\mu_{G}$. The leadinglog expression of the threshold correction (neglecting a small finite correction arising when integrating out the gauge bosons) is given by [24-26]

$$
r_{i}\left(\mu_{G}\right)=\sum_{\xi} b_{i}^{\xi} \ln \left(\frac{m_{\xi}}{\mu_{G}}\right)
$$

which is the same as eq. (2.3) with a replacement $\Lambda \rightarrow \mu_{G}$. The condition of the GCU should be understood as the $i$-independence of the right-hand-side of eq. (A.1). At first glance, the condition seems dependent on the matching scale $\mu_{G}$. However, the condition is independent of $\mu_{G}$ because the $\beta$-function coefficient of the GUT model, $b_{G}$, is related to the MSSM $\beta$-function coefficients by $b_{G}=b_{i}+\sum_{\xi} b_{i}^{\xi}$. The result is illustrated in the left panel of figure 4 . 


\section{A.2 Sequential matchings}

Another way to find the GCU condition is to sequentially construct EFTs and carry out EFT matching every time when the renormalization scale $\mu$ crosses a mass of superheavy particle (see the right panel of figure 4). Let us label the superheavy particles as $\xi_{0}, \xi_{1}, \cdots, \xi_{L}$ with $m_{\xi_{0}}>m_{\xi_{1}}>\cdots>m_{\xi_{L}}$, and assume that the theory is no longer symmetric under the unified gauge group by integrating out $\xi_{0}$. At the scale $\Lambda=m_{\xi_{0}}$, we have the following matching condition

$$
\frac{2 \pi}{\alpha(\Lambda)}=\frac{2 \pi}{\alpha_{i}\left(m_{\xi_{0}}\right)}
$$

The threshold correction arising from integrating out $\xi_{0}$ (as in eq. (A.1)) is vanishing in this case, because the matching scale is taken to be $m_{\xi_{0}}$ and the logarithm vanishes.

Below $\Lambda$, the theory is no longer symmetric under the unified gauge symmetry due to the absence of $\xi_{0}$ and three gauge couplings evolves differently. In particular, the $\beta$ function coefficient is changed from that of the original GUT model, $b_{G}$ ( $i$-independent), to $b_{G}-b_{i}^{\xi_{0}}$ subtracting the contribution from $\xi_{0}$. We run down the three gauge couplings with the new coefficients $b_{G}-b_{i}^{\xi_{0}}$ to the scale $\mu=m_{\xi_{1}}$. The solution to the 1-loop RGE gives us

$$
\frac{2 \pi}{\alpha_{i}\left(m_{\xi_{0}}\right)}=\frac{2 \pi}{\alpha_{i}\left(m_{\xi_{1}}\right)}+\left(b_{G}-b_{i}^{\xi_{0}}\right) \ln \left(\frac{m_{\xi_{1}}}{m_{\xi_{0}}}\right) .
$$

By repeating the same procedure and run down the gauge couplings to $\mu=m_{\xi_{2}}$, we have

$$
\frac{2 \pi}{\alpha_{i}\left(m_{\xi_{1}}\right)}=\frac{2 \pi}{\alpha_{i}\left(m_{\xi_{2}}\right)}+\left(b_{G}-b_{i}^{\xi_{0}}-b_{i}^{\xi_{1}}\right) \ln \left(\frac{m_{\xi_{2}}}{m_{\xi_{1}}}\right) \text {. }
$$

Substituting this to the above equations leads to

$$
\frac{2 \pi}{\alpha(\Lambda)}=\frac{2 \pi}{\alpha_{i}\left(m_{\xi_{2}}\right)}+b_{G} \ln \left(\frac{m_{\xi_{2}}}{\Lambda}\right)-b_{i}^{\xi_{0}} \ln \left(\frac{m_{\xi_{2}}}{m_{\xi_{0}}}\right)-b_{i}^{\xi_{1}} \ln \left(\frac{m_{\xi_{2}}}{m_{\xi_{1}}}\right) .
$$

Repeating the process until the renormalization scale smaller than the lightest superheavy particle mass, we find the relation between the MSSM gauge coupling at $\mu<m_{\xi_{L}}$ and the unified gauge coupling at $\Lambda$ as

$$
\frac{2 \pi}{\alpha(\Lambda)}=\frac{2 \pi}{\alpha_{i}(\mu)}+b_{G} \ln \left(\frac{\mu}{\Lambda}\right)-\sum_{\xi} b_{i}^{\xi} \ln \left(\frac{\mu}{m_{\xi}}\right) .
$$

Since the unified gauge coupling evolves as

$$
\frac{2 \pi}{\alpha(\Lambda)}=\frac{2 \pi}{\alpha_{i}\left(\mu_{G}\right)}+b_{G} \ln \left(\frac{\mu_{G}}{\Lambda}\right)
$$

in the GUT model, eq. (A.7) holds at arbitrary scale around $\mu_{G} \sim 10^{16} \mathrm{GeV}$.

$$
\frac{2 \pi}{\alpha\left(\mu_{G}\right)}=\frac{2 \pi}{\alpha_{i}(\mu)}+b_{G} \ln \left(\frac{\mu}{\mu_{G}}\right)-\sum_{\xi} b_{i}^{\xi} \ln \left(\frac{\mu}{m_{\xi}}\right) .
$$


Here, the condition of GCU is understood as the $i$-independence of the sum of the first and third terms in the right-hand-side. It is apparent that the condition depends neither on $\Lambda$ nor $\mu_{G}$ in this formalism.

It is straightforward to do the similar exercise but evolving gauge couplings in the opposite direction (from low energy to high energy). We find in this case,

$$
\frac{2 \pi}{\alpha\left(\mu_{G}\right)}=\frac{2 \pi}{\alpha_{i}(\mu)}+b_{i} \ln \left(\frac{\mu}{\mu_{G}}\right)+\sum_{\xi} b_{i}^{\xi} \ln \left(\frac{m_{\xi}}{\mu_{G}}\right) .
$$

Eq. (A.9) and (A.10) are of course equivalent with the relation

$$
b_{G}=b_{i}+\sum_{\xi} b_{i}^{\xi}
$$

The combination of the first and second terms in the right-hand-side of eq. (A.10) is nothing but the MSSM gauge couplings at $\mu_{G}$. Thus, it reproduces the previous result eq. (A.1) obtained from the first approach, showing the equivalence between the two formalisms.

\section{A.3 A comment on orbifold GUTs}

In the above two subsections we have seen that the condition of GCU is independent of both the matching scale $\mu_{G}$ and the unification scale $\Lambda$, which appears in the second picture in figure 4 , provided the $\beta$-function coefficients of the MSSM is related to that of the GUT model by $b_{i}=b_{G}+\sum_{\xi} b_{i}^{\xi}$. In the orbifold GUT model, however, the unified gauge symmetry is never realised even at arbitrary high energies in the $4 \mathrm{~d}$ space-time and $b_{G}$ therefore does not exist.

Neglecting a finite correction from the brane kinetic term mentioned in the main text, the following picture is expected [5]. Renormalizing from low energy to high energy, the three gauge couplings of the MSSM approach each other. After passing the compactification scale, $M_{c}, K K$-modes appear and the running changes. In the $\mu>M_{c}$ regime the running is slower but the gauge couplings continue to approach each other. At a scale, $\Lambda$, it is assumed that the three gauge couplings finally meet and the $5 \mathrm{~d}$ theory may be incorporated into a more fundamental theory. Thus, the unification scale $\Lambda$ serves as the cut-off scale of the 5 d theory.

Since there is no unified gauge theory in the $4 \mathrm{~d}$ space-time, the $4 \mathrm{~d}$ EFT matching between the GUT model and its low energy EFT does not make sense, and we are forced to use the bottom-up RGE evolution and eq. (A.10) with $\mu_{G}=\Lambda$. Since eq. (A.10) cannot be related to eq. (A.9), the condition of GCU in orbifold GUTs does depend on $\Lambda$, as can be seen in section 5 .

Open Access. This article is distributed under the terms of the Creative Commons Attribution License (CC-BY 4.0), which permits any use, distribution and reproduction in any medium, provided the original author(s) and source are credited. 


\section{References}

[1] H. Georgi and S.L. Glashow, Unity of All Elementary Particle Forces, Phys. Rev. Lett. 32 (1974) 438 [INSPIRE].

[2] J. Hisano, H. Murayama and T. Yanagida, Probing GUT scale mass spectrum through precision measurements on the weak scale parameters, Phys. Rev. Lett. 69 (1992) 1014 [INSPIRE].

[3] J. Hisano, H. Murayama and T. Yanagida, Nucleon decay in the minimal supersymmetric SU(5) grand unification, Nucl. Phys. B 402 (1993) 46 [hep-ph/9207279] [INSPIRE].

[4] J. Hisano, T. Moroi, K. Tobe and T. Yanagida, Suppression of proton decay in the missing partner model for supersymmetric SU(5) GUT, Phys. Lett. B 342 (1995) 138 [hep-ph/9406417] [INSPIRE].

[5] L.J. Hall and Y. Nomura, Gauge unification in higher dimensions, Phys. Rev. D 64 (2001) 055003 [hep-ph/0103125] [INSPIRE].

[6] D.A. Ross, Threshold Effects in Gauge Theories, Nucl. Phys. B 140 (1978) 1 [INSPIRE].

[7] W.J. Marciano, The Weak Mixing Angle and Grand Unified Gauge Theories, Phys. Rev. D 20 (1979) 274 [INSPIRE].

[8] J.T. Goldman and D.A. Ross, How Accurately Can We Estimate the Proton Lifetime in an SU(5) Grand Unified Model?, Nucl. Phys. B 171 (1980) 273 [inSPIRE].

[9] J.R. Ellis, M.K. Gaillard, D.V. Nanopoulos and S. Rudaz, Uncertainties in the Proton Lifetime, Nucl. Phys. B 176 (1980) 61 [INSPIRE].

[10] H. Murayama and A. Pierce, Not even decoupling can save minimal supersymmetric SU(5), Phys. Rev. D 65 (2002) 055009 [hep-ph/0108104] [InSPIRE].

[11] N. Maekawa and T. Yamashita, Gauge coupling unification in GUT with anomalous U(1) symmetry, Phys. Rev. Lett. 90 (2003) 121801 [hep-ph/0209217] [INSPIRE].

[12] B.S. Acharya, K. Bobkov, G.L. Kane, J. Shao and P. Kumar, The $G_{2}-M S S M$ : An M-theory motivated model of Particle Physics, Phys. Rev. D 78 (2008) 065038 [arXiv:0801.0478] [INSPIRE].

[13] J. Hisano, D. Kobayashi, T. Kuwahara and N. Nagata, Decoupling Can Revive Minimal Supersymmetric SU(5), JHEP 07 (2013) 038 [arXiv:1304.3651] [INSPIRE].

[14] J. Hisano, T. Kuwahara and N. Nagata, Grand Unification in High-scale Supersymmetry, Phys. Lett. B 723 (2013) 324 [arXiv:1304.0343] [INSPIRE].

[15] N. Maekawa and Y. Muramatsu, Nucleon decay via dimension-6 operators in anomalous $\mathrm{U}(1)_{A}$ supersymmetric GUT, Phys. Rev. D 88 (2013) 095008 [arXiv:1307.7529] [inSPIRE].

[16] N. Maekawa and Y. Muramatsu, Nucleon decay via dimension-6 operators in $E_{6} \times \mathrm{SU}(2)_{F} \times \mathrm{U}(1)_{A}$ SUSY GUT model, PTEP 2014 (2014) 113B03 [arXiv:1401.2633] [INSPIRE].

[17] A. Hebecker and J. Unwin, Precision Unification and Proton Decay in F-theory GUTs with High Scale Supersymmetry, JHEP 09 (2014) 125 [arXiv:1405.2930] [INSPIRE].

[18] F. Wang, W. Wang and J.M. Yang, A split SUSY model from SUSY GUT, JHEP 03 (2015) 050 [arXiv: 1501.02906$]$ [INSPIRE]. 
[19] S.A.R. Ellis and J.D. Wells, Visualizing gauge unification with high-scale thresholds, Phys. Rev. D 91 (2015) 075016 [arXiv: 1502.01362] [INSPIRE].

[20] B. Bajc, J. Hisano, T. Kuwahara and Y. Omura, Threshold corrections to dimension-six proton decay operators in non-minimal SUSY SU(5) GUTs, Nucl. Phys. B 910 (2016) 1 [arXiv: 1603.03568] [INSPIRE].

[21] P. Langacker and N. Polonsky, Uncertainties in coupling constant unification, Phys. Rev. D 47 (1993) 4028 [hep-ph/9210235] [INSPIRE].

[22] M. Carena, S. Pokorski and C.E.M. Wagner, On the unification of couplings in the minimal supersymmetric Standard Model, Nucl. Phys. B 406 (1993) 59 [hep-ph/9303202] [INSPIRE].

[23] S. Krippendorf, H.P. Nilles, M. Ratz and M.W. Winkler, Hidden SUSY from precision gauge unification, Phys. Rev. D 88 (2013) 035022 [arXiv:1306.0574] [INSPIRE].

[24] S. Weinberg, Effective Gauge Theories, Phys. Lett. 91B (1980) 51 [inSPIRE].

[25] C.H. LLewellyn Smith, G.G. Ross and J.F. Wheater, Low-energy Predictions From Grand Unified Theories, Nucl. Phys. B 177 (1981) 263 [INSPIRE].

[26] L.J. Hall, Grand Unification of Effective Gauge Theories, Nucl. Phys. B 178 (1981) 75 [INSPIRE].

[27] D. d'Enterria, $\alpha_{s}$ status and perspectives (2018), PoS(DIS2018) 109 (2018) [arXiv: 1806. 06156] [INSPIRE].

[28] S. Pokorski, K. Rolbiecki and K. Sakurai, Proton decay testing low energy supersymmetry with precision gauge unification, Phys. Rev. D 97 (2018) 035027 [arXiv:1707.06720] [INSPIRE].

[29] H. Abe, T. Kobayashi and Y. Omura, Relaxed fine-tuning in models with non-universal gaugino masses, Phys. Rev. D 76 (2007) 015002 [hep-ph/0703044] [INSPIRE].

[30] D. Horton and G.G. Ross, Naturalness and Focus Points with Non-Universal Gaugino Masses, Nucl. Phys. B 830 (2010) 221 [arXiv:0908.0857] [InSPIRE].

[31] A. Kaminska, G.G. Ross and K. Schmidt-Hoberg, Non-universal gaugino masses and fine tuning implications for SUSY searches in the MSSM and the GNMSSM, JHEP 11 (2013) 209 [arXiv: 1308.4168] [INSPIRE].

[32] K. Hagiwara and Y. Yamada, Grand unification threshold effects in supersymmetric SU(5) models, Phys. Rev. Lett. 70 (1993) 709 [INSPIRE].

[33] ATLAS collaboration, Search for squarks and gluinos in final states with jets and missing transverse momentum using $36 \mathrm{fb}^{-1}$ of $\sqrt{\mathrm{s}}=13 \mathrm{TeV}$ pp collision data with the ATLAS detector, Phys. Rev. D 97 (2018) 112001 [arXiv:1712.02332] [InSPIRE].

[34] CMS collaboration, Search for natural and split supersymmetry in proton-proton collisions at $\sqrt{s}=13 \mathrm{TeV}$ in final states with jets and missing transverse momentum, JHEP 05 (2018) 025 [arXiv: 1802.02110] [INSPIRE].

[35] CMS collaboration, Inclusive search for supersymmetry using razor variables in pp collisions at $\sqrt{s}=13 \mathrm{TeV}$, CMS-PAS-SUS-16-017.

[36] ATLAS collaboration, Search for supersymmetry in final states with missing transverse momentum and multiple b-jets in proton-proton collisions at $\sqrt{s}=13$ TeV with the ATLAS detector, ATLAS-CONF-2018-041. 